\title{
DYNAMIC ASSET ALLOCATION AND CONSUMPTION CHOICE IN INCOMPLETE MARKETS
}

\author{
SASHA F. STOIKOV AND THALEIA ZARIPHOPOULOU
}

The University of Texas at Austin

\begin{abstract}
We study the optimal investment and consumption problem of a CRRA investor when the drift and volatility of the stock are driven by a correlated factor. The myopic and non-myopic components of the optimal portfolio process are characterized in terms of the market price of traded and non-traded risk of the minimax martingale measure. We find that the optimal policies depend crucially on the nature of the agent, aggressive versus conservative, and the market incompleteness, improving versus deteriorating investment opportunities. Furthermore, we show that the original problem cannot be decomposed into a pure consumption and a pure terminal wealth problem, unless the market is complete.
\end{abstract}

\section{INTRODUCTION}

The choice of optimal investment strategies and consumption plans is of central interest in financial economics. In complete markets, the powerful duality approach yields the maximal expected utility (value function) via the solution of the dual stochastic optimization problem, which is considerably simpler than the primal one. This methodology is applicable under general assumptions on the asset menu, the evolution of the price processes and the investor's risk preferences (see, among others, Pliska (1986), Cox and Huang (1989), Karatzas et. al. (1987) and the review article by Rogers (2002)). Despite its universality and elegance, duality alone sheds little light on the behavior of the optimal investment and consumption policies in terms of important market inputs, namely, the length of the investment horizon, the market price of traded risk and the investor's risk appetite. These important qualitative questions were first analyzed in Wachter (2002), by assuming that the stock price and the market price of traded risk are perfectly negatively correlated.

Determining the investor's optimal behavior and maximal expected utility becomes a formidable task when the market ceases to be complete. The value function can still be analyzed by duality but, in most cases, the dual problem is not simpler than the original one. General results have been produced by He and Pearson (1991) and Cvitanic and Karatzas (1992) under mild assumptions on prices and market composition (see, also, Kramkov and Schachermayer (1999), Schachermayer (2001), Delbaen et al. (2002), Kabanov and Stricker (2002) and Karatzas

This work was presented at the Mathematical Finance Workshop for Young Researchers, Humboldt University, Berlin, at the Institute of Mathematics and its Applications, Minneapolis and at Imperial College. The authors would like to thank the participants for fruitful comments and suggestions. Stoikov acknowledges partial support from a NSF-VIGRE fellowship and an internship at Foreign Exchange at BNP-Paribas, London. Zariphopoulou acknowledges partial support from grants NSF-DMS 0091946 and NSF-VIGRE 26-1137-01. 
and Zitkovic (2003)). In markets with assets modeled as diffusion processes, the optimal policies can also be produced from the primal problem via the first order conditions in the associated Hamilton-Jacobi-Bellman (HJB) equation; see, among others, Kim and Omberg (1996), Zariphopoulou (2001), Liu (1999), Henderson and Hobson (2002). However, in these works, there is no intermediate consumption, an assumption that considerably facilitates the involved variational arguments. Optimal investment problems with intermediate consumption have been analyzed by Schroder and Skiadas (2003), as well as by Campbell and Viceira (1999) where policies are constructed with the so called log-linear approximation (see, also, Campbell et al. (2004) and Chacko and Viceira (1999)).

Herein, we revisit the optimal investment and consumption problem in the presence of basis risk. We focus on the optimal policies of a small investor in a market environment consisting of a riskless bond and a risky stock. Incompleteness stems from the stochastic nature of the Sharpe ratio of the traded risky asset. Both the latter and its Sharpe ratio are modeled as diffusion processes that are imperfectly correlated. Risk preferences are of Constant Relative Risk Aversion (CRRA) type and the agent's objective is to maximize his/her expected utility of both intermediate consumption and terminal wealth.

In such settings, the optimal portfolio consists of two components, the so-called myopic and non-myopic policies. The myopic policy is the strategy of an investor who ignores what happens beyond the immediate next period. It does not depend on the distribution of asset returns over future revision intervals. The non-myopic investment emerges from the stochasticity of the opportunity set and reflects how the investor reacts to risks that cannot be eliminated. It is also known as the excess risky demand and is the main focus of our study. We express the non-myopic portfolio in terms of the solution of an emerging quasilinear partial differential equation and its first spatial derivative. The solution is also related to the ratio of wealth over optimal consumption. Using this quasilinear equation, we are able to produce analytic results on the size of the optimal policies and their sensitivity in terms of various market inputs.

As expected, it turns out that the agent's optimal behavior is significantly influenced by his/her risk attitude. Quantitatively, this is expressed via the risk aversion parameter $\gamma$ : the investor is said to be aggressive if $\gamma \in(0,1)$ and conservative if $\gamma \in(-\infty, 0)$. We show that aggressive agents implement speculative trading strategies that tend to pay off more than the myopic investment when the investment opportunities improve. Moreover, they behave in accordance to the so-called substitution effect, whereby they consume a smaller proportion of their wealth as investment opportunities improve. On the other hand, conservative investors hedge against worsening stochastic environments by pursuing an investment strategy that tends to pay off more than the myopic investment when the investment opportunities deteriorate. As the investment opportunities improve, they consume a higher proportion of their wealth, a behavior known as the income effect.

To complete the analysis, we also examine the implications of market incompleteness to the additive structure of the primal problem. We recall that in complete markets, the problem of optimal investment and consumption can be split into two sub-problems, namely, one of pure investment and the other of pure consumption. This is a consequence of the linear dual representation of the solution as well as the 
direct link between optimal behavior and hedging of a perfectly replicable underlying risk. We show that if the market is incomplete, the background non-hedgeable risk results in a sub-additive behavior for all investors and for all diffusion dynamics.

The paper is organized as follows. In section 2, we describe the model and give preliminary results on the value function, as well as on special cases of the model. In section 3, we produce the optimal strategies and study the associated quasilinear equation. In section 4 , we provide results on the sensitivity of the optimal policies and numerical results. We conclude with section 5 , where we study the sub-additive behavior of the maximal expected utility.

\section{The MODEL AND ITS MAXIMAL UTILITY}

We consider an optimal investment model for a single agent who manages his/her portfolio by investing in a stock and a riskless bond. The price of the stock $S$ solves

$$
d S_{s}=\mu\left(Y_{s}, s\right) S_{s} d s+\sigma\left(Y_{s}, s\right) S_{s} d W_{s}^{1}
$$

with $S_{t}=S>0$. The process $Y$ will be referred to as the stochastic factor and it is assumed to satisfy

$$
d Y_{s}=b\left(Y_{s}, s\right) d s+a\left(Y_{s}, s\right) d W_{s}
$$

with $Y_{t}=y \in \mathcal{R}$.

The processes $W^{1}$ and $W$ are standard Brownian motions defined on a probability space $\left(\Omega, \mathcal{F},\left(\mathcal{F}_{s}\right), \mathbb{P}\right)$ where $\mathcal{F}_{s}$ is the augmented $\sigma$-algebra. Their correlation coefficient $\rho$ is taken to satisfy $\rho \in(-1,1)$. Assumptions on the drift and diffusion coefficients $\mu, \sigma, a$ and $b$ will be introduced in the sequel. The bond is assumed to offer zero interest rate. The case of (deterministic) non-zero interest rate may be handled by straightforward scaling arguments and is not discussed.

The investor starts at time $t \in[0, T)$ with initial wealth $x \in \mathcal{R}^{+}$. His/her current wealth $X_{s}, t \leq s \leq T$, satisfies the budget constraint $X_{s}=\pi_{s}^{0}+\pi_{s}$ where $\pi_{s}^{0}$ and $\pi_{s}$ are, respectively, the amounts allocated in the bond and the stock accounts. Intermediate consumption is allowed at a (nonnegative) rate $C_{s}, t \leq s \leq T$. Direct calculations involving the dynamics in the above equations yield the evolution of the wealth process

$$
d X_{s}=\mu\left(Y_{s}, s\right) \pi_{s} d s-C_{s} d s+\sigma\left(Y_{s}, s\right) \pi_{s} d W_{s}^{1}
$$

with $X_{t}=x \in \mathcal{R}^{+}$. We denote the Sharpe ratio process of the traded asset by

$$
\lambda_{s}=\lambda\left(Y_{s}, s\right)=\frac{\mu\left(Y_{s}, s\right)}{\sigma\left(Y_{s}, s\right)} .
$$

The investor is endowed with Constant Relative Risk Aversion (CRRA) preferences over consumption and terminal wealth. His/her expected utility payoff is then given by

$$
J(x, y, t ; \pi, C)=E_{\mathbb{P}}\left(\int_{t}^{T} K_{1} \frac{C_{s}^{\gamma}}{\gamma} d s+K_{2} \frac{X_{T}^{\gamma}}{\gamma} \mid X_{t}=x, Y_{t}=y\right)
$$


for $(x, y, t) \in \overline{\mathcal{D}}=\mathcal{R}^{+} \times \mathcal{R} \times[0, T]$, with the processes $Y$ and $X$ solving, respectively, (2) and (3). The coefficients $K_{i}, i=1,2$ are positive constants and will help us analyze the relative contributions of the two utility objectives, namely, the consumption objective in intermediate utility and the wealth objective in the bequest function at terminal time.

The relative risk aversion coefficient $\gamma$ satisfies $\gamma \in(-\infty, 1)$ with $\gamma \neq 0$. The choice $\gamma=0$ corresponds to the logarithmic utility that has been analyzed in Cvitanić and Karatzas (1992) and is not studied herein.

Throughout we will be looking separately at the cases $\gamma \in(0,1)$ and $\gamma \in(-\infty, 0)$. We will be referring to aggressive agents, when $\gamma \in(0,1)$ and to conservative agents, when $\gamma \in(-\infty, 0)$. Aggressive agents have utility that is bounded from below and unbounded from above, while the opposite holds for the conservative ones. The implications of this distinction on the behavior of the two types of investors will be further discussed in Section 4. It is generally accepted that the case of conservative agents is the most relevant empirically (see, among others, Mehra and Prescott (1985), Blake (1996), Palsson (1996), Jacobs (2002), Kaplow (2003)). This class of preferences also gives rise to solutions with better growth and regularity properties (see, for example, Korn and Kraft (2004) and Kraft (2003)).

The wealth process $X$ must satisfy the state constraint

$$
X_{s} \geq 0 \text { a.e } t \leq s \leq T .
$$

This wealth nonnegativity requirement is standard for this class of risk preferences (see, among others, Dybvig and Huang (1988), He and Pearson (1991), Cvitanic and Karatzas (1992), Duffie and Zariphopoulou (1993)).

We next introduce the space $\mathcal{H}=\left\{\left(C_{s}, \pi_{s}\right)\right.$ is $\mathcal{F}_{s}$-measurable with

$E_{\mathbb{P}} \int_{t}^{T} C_{s} d s<\infty$ and $\left.E_{\mathbb{P}} \int_{t}^{T} \sigma^{2}\left(Y_{s}, s\right) \pi_{s}^{2} d s<\infty\right\}$ and the set $\mathcal{A}$ of admissible portfolio and consumption policies, consisting of pairs $(C, \pi) \in \mathcal{H}$ such that the state constraint (6) is satisfied.

The maximal expected utility, also known as value function, is defined as

$$
V(x, y, t)=\sup _{\mathcal{A}} J(x, y, t ; C, \pi) .
$$

The aim herein is to analyze (7) and to construct and study the optimal consumption and portfolio processes, denoted, respectively, by $C_{s}^{*}$ and $\pi_{s}^{*}$. We start with preliminary results on the form of the value function and its regularity. The optimal policies are constructed and analyzed in Sections 3 and 4 .

The following assumptions on the coefficients will be standing throughout:

Assumptions:

i) The market coefficients $\mu, \sigma, a, b: \mathcal{R} \times[0, T] \rightarrow \mathcal{R}$ satisfy the global Lipschitz and linear growth conditions

$$
|f(y, t)-f(\bar{y}, t)| \leq C|y-\bar{y}| \quad \text { and } \quad|f(y, t)| \leq C(1+|y|)
$$

for $t \in[0, T], y, \bar{y} \in \mathcal{R}, C>0$ and $f$ standing for $\mu, \sigma, a$ and $b$.

ii) Uniformly in $y \in \mathcal{R}$ and $t \in[0, T]$, the volatility coefficient $\sigma(y, t)$ satisfies $\sigma(y, t) \geq l>0$ for some constant $l$ and, for fixed $t$, it is an invertible function of $y$.

iii) The market coefficients $\mu, \sigma, a, b: \mathcal{R} \times[0, T] \rightarrow \mathcal{R}$ are bounded and uniformly Hölder continuous functions with $a(y, t)$ satisfying, for some positive constant $\varepsilon$, $a^{2}(y, t) \geq \varepsilon^{2} y^{2}$ for $y \in \mathcal{R}$ and $t \in[0, T]$. 
iv) The market coefficients $\mu, \sigma, a, b: \mathcal{R} \times[0, T] \rightarrow \mathcal{R}$ are such that the solution of (1) satisfies $S_{s}>0$ a.e. for $t \leq s \leq T$ and $S_{t}=S>0$.

The above assumptions are needed for the existence and uniqueness of solutions to (1) and (2), for the wellposedness of the value function (7), and for the uniqueness and regularity properties of the solutions to emerging partial differential equations (see (14) and (17)). They can be relaxed in a number of ways, especially with regards to the requirement that the functions $\mu$ and $\sigma$, and thus the Sharpe ratio of the stock, are bounded. A detailed analysis on the various cases is omitted for the sake of the presentation. We refer the reader to Tiu (2002) for an extensive discussion of the relaxed assumptions on the regularity, growth and ellipticity properties of the relevant market coefficients for the cases of standard and recursive risk preferences.

For the rest of the presentation, we suppress the arguments of the various coefficients and we reinstate them whenever needed. We also introduce the differential operators

$$
\begin{gathered}
\mathcal{L}=\frac{1}{2} a^{2} \frac{\partial^{2}}{\partial y^{2}}+b \frac{\partial}{\partial y}, \\
\mathcal{L}^{\gamma}=\frac{1}{2} a^{2} \frac{\partial^{2}}{\partial y^{2}}+\left(b+\rho \lambda \frac{\gamma}{1-\gamma} a\right) \frac{\partial}{\partial y} .
\end{gathered}
$$

The variational analysis that follows is based on classical arguments for the optimal stochastic control of diffusion processes (see Fleming and Soner (1993), Chapters III and IV). A fundamental ingredient of the theory is that the Dynamic Programming Principle, together with Ito's calculus, yields an important connection between the value function and a fully nonlinear partial differential equation, known as the Hamilton-Jacobi-Bellman equation (see (10) below). This equation is a direct offspring of optimality and stochastic calculus and describes the variational properties of the value function locally in time and space.

If the value function is known to be a priori smooth, classical verification results yield that it is the unique smooth solution of the HJB equation. The first order conditions in the HJB equation can be, in turn, used to produce the optimal policies in the so called feedback form (see Fleming and Soner (1993), Theorem IV.3.1.). In a number of applications however, the value function might not be smooth or, it might not be possible to a priori obtain the required regularity. This is for example the case of stochastic optimization problems with state and control constraints (see (6)), degeneracies, mix of controlled and uncontrolled processes etc. In this case, the notion of solution to the HJB equation needs to be relaxed. It turns out that the appropriate class of solutions, in which we obtain strong uniqueness, existence and robustness results, is the one of viscosity solutions.

The notion of viscosity solution was introduced by Crandall and Lions (1983) for first order equations, and by Lions (1983) for second order equations. For a general overview of the theory of viscosity solutions, we refer to the User's Guide of Crandall et. al. (1992). When state constraints are binding, as it is the case herein, the notion of constrained viscosity solutions is used. These solutions were introduced by Soner (1986) and Capuzzo-Dolcetta and Lions (1987) for first order equations (see also, Ishii and Lions (1990), Katsoulakis (1994), Barles (1994)). 
In stochastic control problems arising in models of optimal investment and consumption, the use of (constrained) viscosity solutions was initiated by Zariphopoulou (1989) (see, also, Fleming and Zariphopoulou (1991) and Zariphopoulou (1992)). These solutions were subsequently used by others (Shreve and Soner (1994), Duffie and Zariphopoulou (1993), Davis et al. (1993)) and by now, they have become a standard tool for the analysis of maximal expected utility problems and other stochastic control problems arising in Mathematical Finance (see, among others, El Karoui and Quenez (1997), Soner and Touzi (2002), Bouchard (2002), Pang (2004)).

There are two main advantages for using this family of weak solutions. First, they provide a rigorous characterization of the value function as the unique (constrained) viscosity solution of the HJB equation. This is in turn used for the construction of the value function itself, if a suitable candidate can be found (see Propositions 2 and 3). The second advantage is that, when explicit solutions cannot be found and numerical approximations are sought, they yield strong convergence results for the involved numerical schemes (see, Davis et al. (1993), Tourin and Zariphopoulou (1994), Fitzpatrick and Fleming (1990), Bouchard et. al. (2004), Cont et. al. (2004)).

In what follows we state a uniqueness result for the value function in the constrained viscosity sense. Given the interests of the audience and in order to facilitate the exposition, we do not provide detailed technical arguments. Instead, we state the definition of constrained viscosity solutions in Appendix A and give therein an outline of the proof and references for the omitted steps. For a detailed exposition of the technical arguments, we refer the reader to the survey papers of Soner (1997), Zariphopoulou (2003) and Touzi (2004).

\section{i) The Hamilton-Jacobi-Bellman equation and reduced form solutions}

The first result gives the general characterization of the value function as the appropriate solution of the associated Hamilton-Jacobi-Bellman (HJB) equation.

Proposition 1. The value function is a constrained viscosity solution on $\bar{D}$ of the Hamilton-Jacobi-Bellman equation

$$
\begin{aligned}
V_{t}+ & \max _{\pi}\left(\frac{1}{2} \sigma^{2} \pi^{2} V_{x x}+\pi\left(\rho \sigma a V_{x y}+\mu V_{x}\right)\right) \\
& +\max _{c}\left(-c V_{x}+K_{1} \frac{c^{\gamma}}{\gamma}\right)+\mathcal{L} V=0
\end{aligned}
$$

and

$$
V(x, y, T)=K_{2} \frac{x^{\gamma}}{\gamma}
$$

with $\mathcal{L}$ as in (8). Moreover, it is the unique such solution in the class of functions that are concave and non decreasing in $x$, and, for fixed $(x, t)$, bounded in $y$.

From the above theorem we deduce that if we find a solution to the HJB equation that is smooth and satisfies the appropriate variational constraint at the boundary of the domain $\overline{\mathcal{D}}$, then it coincides with the value function. This follows because, on the one hand, this smooth solution is automatically a constrained viscosity solution of the HJB equation and, on the other hand, the value function is the unique such solution. 
Next, we construct such a candidate which we denote, by a slight abuse of notation, by $V$. To this end, using the homogeneity properties of the utility payoff (5) and standard arguments (see Merton (1969)) we first observe that it must be of the separable form

$$
V(x, y, t)=\frac{x^{\gamma}}{\gamma} F(y, t) \quad \text { for } \quad(x, y, t) \in \overline{\mathcal{D}} .
$$

The component $F$ solves a simpler equation than the HJB one, namely, a quasilinear equation (i.e. the equation remains linear with respect to the second order derivative). However, $F$ cannot be obtained in closed form unless further model assumptions are made. If the market is complete, a change of unknown transformation linearizes the reduced equation, a fact well established and consistent with the duality approach. If the market is incomplete, the reduced equation can be still linearized, but only if there is no intermediate consumption and, at the same time, low dimensionality. In general, linearization is not possible but transformations of $F$ are often used because they provide natural representations of quantities of interest. Such transformations will be referred to as distortions (see, Zariphopoulou (2001) and Monoyios (2004)).

As the analysis below shows, there are two appropriate distortion representations of the component $F$. The first one is presented in Proposition 2 and depends on the risk aversion and the correlation (see (12) and (13)). The emerging equation turns out to be of reaction-diffusion type (see (14)). Such reaction-diffusion equations appeared for the first time in Tiu (2002) who analyzed a similar optimal investment and consumption model but allowed for more general risk preferences, namely, for recursive ones (of Kreps-Porteus and Uzawa type). This work was recently extended to a partial information model setting by Tiu (2004). For the pure investment problem $\left(K_{1}=0\right)$, the nonlinear term disappears, a special case which we describe in detail in subsection (ii) (see, also Zariphopoulou (1999), (2001) and Tehranchi (2004)).

The second distortion transformation, which we present in Proposition 3, does not depend on the risk aversion. In the special case of complete markets $\left(\rho^{2}=\right.$ 1), which we analyze in subsection (iii), the emerging equation turns out to be linear. This distortion has been used extensively in complete markets (see, among others, Merton (1969), Karatzas et. al. (1987), Zariphopoulou (1999) and Wachter (2002)), since it yields an important connection between the components of the value function and the wealth to consumption ratio. In Section 3 we will prove that this connection is valid even if the market ceases to be complete.

Naturally, as the market becomes complete, i.e. as $\rho^{2} \rightarrow 1$, the two distortion transformations, (13) and (16), reduce to the same expression (see Proposition 6).

We provide the two value function representations below.

Proposition 2. Let $\lambda, \mathcal{L}^{\gamma}$ and $\delta$ be, respectively the Sharpe ratio (4), the operator (9) and the distortion power

$$
\delta=\frac{1-\gamma}{1-\gamma+\rho^{2} \gamma}
$$

The value function $V$ is given by

$$
V(x, y, t)=\frac{x^{\gamma}}{\gamma} v(y, t)^{\delta}
$$


where $v: \mathcal{R} \times[0, T] \rightarrow \mathcal{R}^{+}$is the unique $C^{2,1}(\mathcal{R} \times[0, T])$ solution of the reactiondiffusion equation

$$
v_{t}+\mathcal{L}^{\gamma} v+\frac{\gamma}{2 \delta(1-\gamma)} \lambda^{2} v+K_{1}^{1 /(1-\gamma)}\left(1-\gamma+\rho^{2} \gamma\right) v^{p}=0
$$

with

$$
p=\frac{-\gamma\left(1-\rho^{2}\right)}{1-\gamma+\rho^{2} \gamma}
$$

and $v(y, T)=K_{2}^{1 / \delta}$.

Proposition 3. Let $\lambda$ and $\mathcal{L}^{\gamma}$ be, respectively, the Sharpe ratio (4) and the operator (9). The value function $V$ is given by

$$
V(x, y, t)=\frac{x^{\gamma}}{\gamma} h(y, t)^{1-\gamma}
$$

where $h: \mathcal{R} \times[0, T] \rightarrow \mathcal{R}^{+}$is the unique $C^{2,1}(\mathcal{R} \times[0, T])$ solution of the quasilinear equation

$$
h_{t}+\mathcal{L}^{\gamma} h+\frac{\gamma}{2(1-\gamma)^{2}} \lambda^{2} h-\frac{1}{2} \gamma\left(1-\rho^{2}\right) a^{2} \frac{h_{y}^{2}}{h}+K_{1}^{1 /(1-\gamma)}=0
$$

with $h(y, T)=K_{2}^{1 / 1-\gamma}$.

Formulae (13), (16) and equations (14), (17) can be verified by direct substitution in the HJB equation and the rest of the proofs follows from the uniqueness properties of the value function.

Note that the claimed regularity of $v$, and in turn of $h$, is not immediate. Indeed, the classical results for solutions of reaction-diffusion equations (see, among others, Rothe (1984) and Smoller (1994)) cannot be directly applied due to the non-Lipschitz regularity of the reaction-diffusion term for certain values of the risk aversion parameter. The aforementioned regularity was established by Tiu (2002, Theorem 4.2).

ii) Utility from terminal wealth under imperfect correlation

In the absence of intermediate consumption, the wealth process, $X^{w}$, satisfies

$$
d X_{s}^{w}=\mu\left(Y_{s}, s\right) \pi_{s}^{w} d s+\sigma\left(Y_{s}, s\right) \pi_{s}^{w} d W_{s}^{1}
$$

with the set of admissible policies defined as $\mathcal{A}^{w}=\left\{\pi_{s}^{w}: \pi_{s}^{w}\right.$ is $\mathcal{F}_{s}$ - measurable,

$E_{\mathbb{P}} \int_{t}^{T} \sigma^{2}\left(Y_{s}, s\right)\left(\pi_{s}^{w}\right)^{2} d s<\infty$ and $X_{s}^{w} \geq 0$ a.e. $\}$. The value function, $V^{w}$, is given by

$$
V^{w}(x, y, t)=\sup _{\mathcal{A}^{w}} E_{\mathbb{P}}\left(K_{2} \frac{\left(X_{T}^{w}\right)^{\gamma}}{\gamma} \mid X_{t}^{w}=x, Y_{t}=y\right)
$$

for $(x, y, t) \in \overline{\mathcal{D}}$. As in the general case, it is expected that $V^{w}$ is represented in the separable form (11). Due to the absence of intermediate consumption, the factor $F$ can be obtained in closed form.

To facilitate the exposition of the representation results, we introduce the measure $\mathbb{P}^{\gamma}$. We caution the reader that $\mathbb{P}^{\gamma}$ is not a martingale measure since, under it, the stock price process fails to be a martingale. 
Definition 4. Let $\mathbb{P}^{\gamma}$ be the measure defined by its Radon-Nikodym derivative

$$
\frac{d \mathbb{P}^{\gamma}}{d \mathbb{P}}=\exp \left(-\int_{0}^{T} \frac{\gamma}{\gamma-1} \lambda\left(Y_{s}, s\right) d W_{s}^{1}-\int_{0}^{T} \frac{1}{2} \frac{\gamma^{2}}{(\gamma-1)^{2}} \lambda\left(Y_{s}, s\right)^{2} d s\right)
$$

Under $\mathbb{P}^{\gamma}$, the processes $W^{1, \gamma}$ and $W^{\gamma}$, defined by

$$
d W_{s}^{1, \gamma}=d W_{s}^{1}+\frac{\gamma}{\gamma-1} \lambda\left(Y_{s}, s\right) d s
$$

and

$$
d W_{s}^{\gamma}=d W_{s}+\rho \frac{\gamma}{\gamma-1} \lambda\left(Y_{s}, s\right) d s
$$

are standard Brownian motions. The dynamics of the stock and the stochastic factor under $\mathbb{P}^{\gamma}$ are then given by

$$
d S_{s}=\frac{\mu\left(Y_{s}, s\right)}{1-\gamma} S_{s} d s+\sigma\left(Y_{s}, s\right) S_{s} d W_{s}^{1, \gamma}
$$

and

$$
d Y_{s}=\left(b\left(Y_{s}, s\right)+\rho \frac{\gamma}{1-\gamma} \lambda\left(Y_{s}, s\right) a\left(Y_{s}, s\right)\right) d s+a\left(Y_{s}, s\right) d W_{s}^{\gamma}
$$

The following result was established in Zariphopoulou (2001, Theorem 3.2).

Proposition 5. The value function $V^{w}$ of the pure investment problem (19) is given by

$$
V^{w}(x, y, t)=\frac{x^{\gamma}}{\gamma} v^{w}(y, t)^{\delta}
$$

where $v^{w}$ solves

$$
v_{t}^{w}+\mathcal{L}^{\gamma} v^{w}+\frac{1}{2} \frac{\gamma}{\delta(1-\gamma)} \lambda^{2} v^{w}=0
$$

with $v^{w}(y, T)=K_{2}^{1 / \delta}$ and $\delta$ as in (12). It admits the probabilistic representation

$$
V^{w}(x, y, t)=K_{2} \frac{x^{\gamma}}{\gamma}\left(E_{\mathbb{P}^{\gamma}}\left(M(t, T)^{1 / \delta} \mid Y_{t}=y\right)\right)^{\delta}
$$

where $\mathbb{P}^{\gamma}$ is defined in (20) and

$$
M(t, s)=e^{\int_{t}^{s} \frac{\gamma}{2(1-\gamma)} \lambda^{2}\left(Y_{u}, u\right) d u} \quad \text { for } \quad t \leq s \leq T,
$$

with $Y$ solving (21).

Proof. Equation (23) follows from (14) if we set $K_{1}=0$ (recall that $\gamma<1$ ), a choice that corresponds to the absence of utility from intermediate consumption. The probabilistic representation (24) is a direct consequence of the Feynman-Kac formula, the definition of $\mathbb{P}^{\gamma}$ and the form of the dynamics of the stochastic factor process under it.

iii) Utility from terminal wealth and intermediate consumption under perfect correlation

The complete market case may be conveniently modeled via a setting of nonlinear stock dynamics. To simplify the exposition, we only consider the case $\rho=1$. We 
may then assume that the stochastic factor represents the stock through $Y_{s} \equiv S_{s}$, $b(y, t) \equiv \mu(y, t) y$ and $a \equiv \sigma(y, t) y$, or equivalently, that the stock solves

$$
d \tilde{S}_{s}=\mu\left(\tilde{S}_{s}, s\right) \tilde{S}_{s} d s+\sigma\left(\tilde{S}_{s}, s\right) \tilde{S}_{s} d \tilde{W}_{s},
$$

where $\tilde{W}$ is a standard Brownian motion on a probability space, say $(\tilde{\Omega}, \tilde{\mathcal{F}}, \tilde{\mathbb{P}})$.

The wealth process $\tilde{X}$ satisfies

$$
d \tilde{X}_{s}=\mu\left(\tilde{S}_{s}, s\right) \tilde{\pi}_{s} d s-\tilde{C}_{s} d s+\sigma\left(\tilde{S}_{s}, s\right) \tilde{\pi}_{s} d \tilde{W}_{s},
$$

while the value function $\tilde{V}$ is given by

$$
\tilde{V}(x, S, t)=\sup _{\mathcal{A}} E_{\tilde{\mathbb{P}}}\left(\int_{t}^{T} K_{1} \frac{\tilde{C}_{s}^{\gamma}}{\gamma} d s+K_{2} \frac{\tilde{X}_{T}^{\gamma}}{\gamma} \mid \tilde{X}_{t}=x, \tilde{S}_{t}=S\right)
$$

with the set of admissible policies $\mathcal{A}$ appropriately defined.

We easily deduce that setting $\rho=1, a(y, t)=\sigma(y, t) y$ and $b(y, t)=\mu(y, t) y$ in (17), yields, in combination with (16), the value function representation

$$
\tilde{V}(x, S, t)=\frac{x^{\gamma}}{\gamma} \tilde{h}(S, t)^{1-\gamma}
$$

with $\tilde{h}$ solving

$$
\tilde{h}_{t}+\frac{1}{2} \sigma^{2} S^{2} \tilde{h}_{S S}+\frac{\mu}{1-\gamma} S \tilde{h}_{S}+\frac{\gamma}{2(1-\gamma)^{2}} \lambda^{2} \tilde{h}+K_{1}^{1 /(1-\gamma)}=0
$$

and $\tilde{h}(S, T)=K_{2}^{1 /(1-\gamma)}$.

The value function formula (29), presented below, may be found in Zariphopoulou (1999); see, also Wachter (2002) for a special case and Karatzas et. al. (1987) for general diffusion dynamics.

Proposition 6. Let $\tilde{\mathbb{P}}^{\gamma}$ be a measure defined by its Radon-Nikodym derivative

$$
\frac{d \tilde{\mathbb{P}}^{\gamma}}{d \tilde{\mathbb{P}}}=\exp \left(-\int_{0}^{T} \frac{\gamma}{\gamma-1} \lambda\left(S_{s}, s\right) d W_{s}^{1}-\int_{0}^{T} \frac{1}{2} \frac{\gamma^{2}}{(\gamma-1)^{2}} \lambda\left(S_{s}, s\right)^{2} d s\right) .
$$

The value function $\tilde{V}$ of the complete market problem (27) is given by

$$
\tilde{V}(x, S, t)=\frac{x^{\gamma}}{\gamma} \tilde{h}(S, t)^{1-\gamma}
$$

where $\tilde{h}$ solves (28). It admits the probabilistic representation

$$
\begin{gathered}
\tilde{V}(x, S, t)=\frac{x^{\gamma}}{\gamma} E_{\tilde{\mathbb{P}} \gamma}\left(\int_{t}^{T} \tilde{M}(t, s)^{1 /(1-\gamma)} K_{1}^{1 /(1-\gamma)} d s\right. \\
\left.+\tilde{M}(t, T)^{1 /(1-\gamma)} K_{2}^{1 /(1-\gamma)} \mid \tilde{S}_{t}=S\right)^{1-\gamma}
\end{gathered}
$$

where

$$
\tilde{M}(t, s)=e^{\int_{t}^{s} \frac{\gamma}{2(1-\gamma)} \lambda^{2}\left(\tilde{S}_{u}, u\right) d u} \quad \text { for } \quad t \leq s \leq T
$$

with $\tilde{S}$ solving (26). 


\section{iv) Uniform bounds on the value function}

We finish this section by presenting some uniform bounds on the value function. The technical arguments are based on the so-called comparison principle for the associated partial differential equations which enables us to bound the solution $V$ by appropriately chosen sub- and super-solutions. The proof is presented in Appendix A.

Proposition 7. Let $\mathbb{P}^{\gamma}$ be the measure defined in (20) and $M$ as in (25).

i) If the investor is aggressive, i.e. $\gamma \in(0,1)$, then, the value function satisfies, for $(x, y, t) \in \overline{\mathcal{D}}$,

$$
V(x, y, t) \geq K_{2} \frac{x^{\gamma}}{\gamma}\left(E_{\mathbb{P} \gamma}\left(M(t, T)^{1 / \delta} \mid Y_{t}=y\right)\right)^{\delta}
$$

and

$$
V(x, y, t) \leq \frac{x^{\gamma}}{\gamma} \check{H}(y, t)^{1-\gamma}
$$

where

$\check{H}(y, t)=E_{\mathbb{P} \gamma}\left(\int_{t}^{T} M(t, s)^{1 /(1-\gamma)} K_{1}^{1 /(1-\gamma)} d s+M(t, T)^{1 /(1-\gamma)} K_{2}^{1 /(1-\gamma)} \mid Y_{t}=y\right)$

ii) If the investor is conservative, i.e. $\gamma \in(-\infty, 0)$, then

$$
V(x, y, t) \leq K_{2} \frac{x^{\gamma}}{\gamma}\left(E_{\mathbb{P} \gamma}\left(M(t, T)^{1 / \delta} \mid Y_{t}=y\right)\right)^{\delta}
$$

and

$$
V(x, y, t) \leq \frac{x^{\gamma}}{\gamma} \check{H}(y, t)^{1-\gamma}
$$

\section{Optimal CONSUMPtion AND INVESTMENT STRATEGIES}

In this section we construct and study the optimal consumption and investment policies, denoted respectively, by $C_{s}^{*}$ and $\pi_{s}^{*}$. They are derived in the so-called feedback form

$$
C_{s}^{*}=C^{*}\left(X_{s}^{*}, Y_{s}, s\right) \quad \text { and } \pi_{s}^{*}=\pi^{*}\left(X_{s}^{*}, Y, s\right)
$$

with the feedback functions $C^{*}: \mathcal{D} \rightarrow \mathcal{R}^{+}$and $\pi^{*}: \mathcal{D} \rightarrow \mathcal{R}$ depending solely on the current states of the optimal wealth and the stochastic factor. The functionals $C^{*}$ and $\pi^{*}$ are determined analytically via the first order conditions in the HJB equation (10). This construction of optimal policies is standard and optimality is established from classical verification theorems (see Fleming and Soner (1993), Theorem IV.3.1 and Corollary IV.3.1). The key steps are given in Appendix B.

Proposition 8. Let $Y$ be the stochastic factor solving (2), $h$ the solution of (17) and $X^{*}$ solving (42). Then, the optimal consumption and investment policies, $C_{s}^{*}$ and $\pi_{s}^{*}$, are given, for $t \leq s \leq T$, in the feedback form

$$
C_{s}^{*}=C^{*}\left(X_{s}^{*}, Y_{s}, s\right)=K_{1}^{1 /(1-\gamma)} \frac{X_{s}^{*}}{h\left(Y_{s}, s\right)}
$$


and

$$
\pi_{s}^{*}=\pi^{*}\left(X_{s}^{*}, Y_{s}, s\right)=\pi_{s}^{m}+H_{s}
$$

where

$$
\pi_{s}^{m}=\pi^{m}\left(X_{s}^{*}, Y_{s}, s\right)=\lambda\left(Y_{s}, s\right) \frac{X_{s}^{*}}{(1-\gamma) \sigma\left(Y_{s}, s\right)}
$$

is the myopic component and

$$
H_{s}=H\left(X_{s}^{*}, Y_{s}, s\right)=\rho a\left(Y_{s}, s\right) \frac{h_{y}\left(Y_{s}, s\right)}{h\left(Y_{s}, s\right)} \frac{X_{s}^{*}}{\sigma\left(Y_{s}, s\right)}
$$

is the non-myopic component of the investment policy. The investor's optimal wealth $X_{s}^{*}, t \leq s \leq T$, solves

$$
X_{s}^{*}=x+\int_{t}^{s} \mu\left(Y_{u}, u\right) \pi_{u}^{*}-C_{u}^{*} d u+\int_{t}^{s} \sigma\left(Y_{u}, u\right) \pi_{u}^{*} d W_{u}^{1}
$$

while the value function $V$ is given, for $(x, y, t) \in \overline{\mathcal{D}}$, by

$$
V(x, y, t)=E_{\mathbb{P}}\left(\int_{t}^{T} K_{1} \frac{\left(C_{s}^{*}\right)^{\gamma}}{\gamma} d s+K_{2} \frac{\left(X_{T}^{*}\right)^{\gamma}}{\gamma} \mid X_{t}^{*}=x, Y_{t}=y\right) .
$$

The above functional dependencies enable us to express the non-myopic component of the optimal portfolio $H_{s}$ in terms of the optimal consumption process.

Corollary 9. If $C_{s}^{*}=C^{*}\left(X_{s}^{*}, Y_{s}, s\right)$ is the optimal consumption process then the optimal portfolio component $H_{s}$ satisfies

$$
H_{s}=-\rho \frac{a\left(Y_{s}, s\right)}{\sigma\left(Y_{s}, s\right)} \frac{\partial}{\partial y} \ln C^{*}\left(X_{s}^{*}, Y_{s}, s\right)=-\rho \frac{a\left(Y_{s}, s\right)}{\sigma\left(Y_{s}, s\right)} \frac{C_{y}^{*}\left(X_{s}^{*}, Y_{s}, s\right)}{C^{*}\left(X_{s}^{*}, Y_{s}, s\right)} .
$$

The main interest for the rest of this section is in the interpretation and representation of the optimal investment and consumption. Probabilistic results for these policies have been produced with duality arguments under very mild model assumptions (see, among others, He and Pearson (1991), Cvitanic and Karatzas (1992)). Through the dual problem, the optimal consumption process is provided in closed form but not the optimal investment. Existence results for the latter are provided via martingale representation results for certain stochastic integrals emerging in the dual domain. To our knowledge, however, no closed-form or explicit results have been produced for the optimal investments. Herein, we use the scaling properties of the value function, together with the variational properties of the value function component $h$, see (17), to provide such results.

As formula (39) shows the optimal portfolio consists of two investment components, namely, the myopic and the non-myopic one. This well established result is a direct consequence of the stochasticity of the investment opportunity set (see, for example, Kim and Omberg (1996), Campbell and Viceira (1999) and Liu (1999)). The myopic policy neglects the stochastic evolution of the Sharpe ratio $\lambda_{s}=\lambda\left(Y_{s}, s\right)$ and its form is consistent with the policy followed by the investor under a, locally in time, non-stochastic Sharpe ratio. The non-myopic component, however, yielding the excess demand for the risky asset, comes from the stochastic evolution of the process $Y$. It reflects how the investor should act in order to manage the risk 
generated by the stochastic factor. This risk will be represented via a process, denoted by $\lambda_{s}^{\perp, *}$, that appears in the density of an appropriately chosen equivalent martingale measure (see Proposition 10 and Theorem 11).

We will naturally refer to the two processes, $\lambda_{s}$ and $\lambda_{s}^{\perp, *}$, as the market prices of traded and non-traded risk. The important observation is that the optimal portfolio is given as a linear combination of the two types of risk (see (51) and (52)) with the linearity parameter depending on the degree of market incompleteness, as expressed through the correlation between the stock price and the stochastic factor. We stress that the process $\lambda_{s}^{\perp, *}$ has already emerged in various duality works and that the decomposition result (52) is not entirely new. It can be found in dual formulae appearing, among others, in Monoyios (2004) and Hobson (2004) in settings without intermediate consumption. Herein, we derive it by solving the primal problem and we focus on its variational rather than its dual properties.

To facilitate the exposition, we start with the characterization of a relevant equivalent martingale measure. We recall that if $\mathcal{Q}_{e}$ is the set of equivalent martingale measures, the density of its generic element, say $Q$, can be represented as

$$
\frac{d Q\left(\lambda, \lambda^{\perp}\right)}{d \mathbb{P}}=\exp \left(-\int_{0}^{T} \lambda_{s} d W_{s}^{1}-\int_{0}^{T} \lambda_{s}^{\perp} d W_{s}^{1, \perp}-\int_{0}^{T} \frac{1}{2}\left(\lambda_{s}^{2}+\left(\lambda_{s}^{\perp}\right)^{2}\right) d s\right)
$$

where $\lambda_{s}$ is the stock's Sharpe ratio, $W^{1, \perp}$ is a standard Brownian motion orthogonal to $W^{1}$ under $\mathbb{P}$ and $\lambda_{s}^{\perp}$ is a parameter process.

In the sequel, we will be using the exponential process

$$
\left.\frac{d Q\left(\lambda, \lambda^{\perp}\right)}{d \mathbb{P}}\right|_{t} ^{s}=\exp \left(-\int_{t}^{s} \lambda_{u} d W_{u}^{1}-\int_{t}^{s} \lambda_{u}^{\perp} d W_{u}^{1, \perp}-\int_{t}^{s} \frac{1}{2}\left(\lambda_{u}^{2}+\left(\lambda_{u}^{\perp}\right)^{2}\right) d u\right)
$$

to define the dual expected criterion

$$
\begin{gathered}
\mathcal{H}(Q ; \mathbb{P})=E_{\mathbb{P}}\left(\int_{0}^{T}\left(\left.\frac{d Q}{d \mathbb{P}}\right|_{0} ^{s}\right)^{\gamma /(\gamma-1)} K_{1}^{1 /(1-\gamma)} d s\right. \\
\left.+\left(\left.\frac{d Q}{d \mathbb{P}}\right|_{0} ^{T}\right)^{\gamma /(\gamma-1)} K_{2}^{1 /(1-\gamma)} \mid \mathcal{F}_{0}\right)
\end{gathered}
$$

for $Q \in \mathcal{Q}_{e}$.

We also consider the optimization problems

$$
\mathcal{H}\left(\mathbb{Q}^{\text {min }} ; \mathbb{P}\right)=\inf _{Q \in \mathcal{Q}_{e}} \mathcal{H}(Q ; \mathbb{P}) \quad \text { for } \quad \gamma \in(0,1)
$$

and

$$
\mathcal{H}\left(\mathbb{Q}^{\max } ; \mathbb{P}\right)=\sup _{Q \in \mathcal{Q}_{e}} \mathcal{H}(Q ; \mathbb{P}) \quad \text { for } \quad \gamma \in(-\infty, 0) .
$$

The above expected criteria are related to the so-called minimax equivalent martingale measures introduced by He and Pearson (1991). The existence of optimizers in a general semimartingale setting has been studied and established by Kramkov and Schachermayer (1999) and Bellini and Fritelli (2002).

Below, we relate these optimizers with the spatial derivatives of the function $h$ appearing in the primal representation of the value function (see (17)). To our knowledge, this representation appears to be new. 
Proposition 10. Let the agent be aggressive, i.e. $\gamma \in(0,1)$, and let $\mathbb{Q}^{\text {min }}$ be the optimizer of

$$
\mathcal{H}\left(\mathbb{Q}^{\text {min }} ; \mathbb{P}\right)=\inf _{Q \in \mathcal{Q}_{e}} \mathcal{H}(Q ; \mathbb{P})
$$

Respectively, for conservative agents, i.e. for $\gamma \in(-\infty, 0)$, let $\mathbb{Q}^{\text {max }}$ be the optimizer of

$$
\mathcal{H}\left(\mathbb{Q}^{\max } ; \mathbb{P}\right)=\sup _{Q \in \mathcal{Q}_{e}} \mathcal{H}(Q ; \mathbb{P})
$$

Then, the associated measures $\mathbb{Q}^{\text {min }}$ and $\mathbb{Q}^{\text {max }}$ are represented by

$$
\frac{d \mathbb{Q}}{d \mathbb{P}}=\exp \left(-\int_{0}^{T} \lambda_{s} d W_{s}^{1}-\int_{0}^{T} \lambda_{s}^{\perp, *} d W_{s}^{1, \perp}-\int_{0}^{T} \frac{1}{2}\left(\lambda_{s}^{2}+\left(\lambda_{s}^{\perp, *}\right)^{2}\right) d s\right)
$$

(cf. (44)) with the optimal parameter process $\lambda_{s}^{\perp, *}$ given by

$$
\lambda_{s}^{\perp, *}=\lambda^{\perp, *}\left(Y_{s}, s\right)
$$

with $\lambda^{\perp, *}: \mathcal{R} \times[0, T] \rightarrow \mathcal{R}$ satisfying

$$
\lambda^{\perp, *}(y, t)=-(1-\gamma) \sqrt{1-\rho^{2}} a(y, t) \frac{h_{y}(y, t)}{h(y, t)}
$$

for $h$ solving (17).

Reconciling (39), (40), (41) and (50) yields the following decomposition result.

Theorem 11. Let $\lambda_{s}$ and $\lambda_{s}^{\perp, *}$ be, respectively, the Sharpe ratio (4) and the optimizer (50).

i) The optimal investment $\pi_{s}^{*}$ is given by

$$
\pi_{s}^{*}=\Lambda_{s} \frac{X_{s}^{*}}{\sigma\left(Y_{s}, s\right)(1-\gamma)}
$$

where

$$
\Lambda_{s}=\lambda_{s}-\frac{\rho}{\sqrt{1-\rho^{2}}} \lambda_{s}^{\perp, *},
$$

where $X^{*}$ is the CRRA agent's optimal wealth, solving (42), and $\rho$ the correlation between the stock price $S$ and the stochastic factor $Y$.

ii) The myopic and non-myopic investment process $\pi_{s}^{m}$ and $H_{s}$ are given, respectively, by

$$
\pi_{s}^{m}=\lambda_{s} \frac{X_{s}^{*}}{\sigma\left(Y_{s}, s\right)(1-\gamma)}
$$

and

$$
H_{s}=-\lambda_{s}^{\perp, *} \frac{\rho}{\sqrt{1-\rho^{2}}} \frac{X_{s}^{*}}{\sigma\left(Y_{s}, s\right)(1-\gamma)} .
$$

The above result provides an intuitively pleasing way to characterize the optimal excess demand for the risky asset. The aggressive (resp. conservative) investor's first task is to choose the optimal measure $\mathbb{Q}^{\min }\left(\lambda_{s}^{\perp, *}\right)$ (resp. $\left.\mathbb{Q}^{\max }\left(\lambda_{s}^{\perp, *}\right)\right)$. In other words, given his/her risk preferences and the market incompleteness, the agent assigns the market price of non-traded risk associated with the non-traded factor $Y$. The optimal investment behavior is then completely characterized by 
the traded and non-traded market prices of risk via the linear structural result, as expressed in (51) and (52).

This representation for the optimal investment strategy may be interpreted as the myopic strategy under a modified Sharpe ratio, given by $\Lambda_{s}$, which accounts for the perceived effect of the stochastic factor. For instance, let us assume that the market price of non-traded risk $\lambda_{s}^{\perp, *}$ is positive. If the stock $S$ and the stochastic factor $Y$ are positively correlated, the presence of $Y$ has the perceived effect of enhancing the riskiness of the stock. In such a setting, the demand for the risky asset is lower than the myopic demand, i.e. $\pi_{s}^{*}<\pi_{s}^{m}$. On the other hand, if $S$ and $Y$ are negatively correlated, the factor $Y$ dampens the stock's degree of riskiness and, therefore, the investor will hold more than the myopic amount.

This intuition is reversed when the market price of non-traded risk $\lambda_{s}^{\perp \text {,* }}$ is negative. Of course, when the correlation coefficient is zero, the investor holds exactly the myopic portfolio even in the presence of a stochastic opportunity set.

Remark: In the absence of intermediate consumption (i.e. when $K_{1}=0$ ), the dual criteria (47) and (48) simplify to

$$
\mathcal{H}\left(\mathbb{Q}^{\text {min }} ; \mathbb{P}\right)=K_{2}^{1 /(1-\gamma)} \inf _{Q \in \mathcal{Q}_{e}} E_{\mathbb{P}}\left[\left(\frac{d Q\left(\lambda, \lambda^{\perp}\right)}{d \mathbb{P}}\right)^{\frac{\gamma}{1-\gamma}}\right] \quad \text { for } \quad \gamma \in(0,1)
$$

and

$$
\mathcal{H}\left(\mathbb{Q}^{\max } ; \mathbb{P}\right)=K_{2}^{1 /(1-\gamma)} \sup _{Q \in \mathcal{Q}_{e}} E_{\mathbb{P}}\left[\left(\frac{d Q\left(\lambda, \lambda^{\perp}\right)}{d \mathbb{P}}\right)^{\frac{\gamma}{1-\gamma}}\right] \quad \text { for } \quad \gamma \in(-\infty, 0) .
$$

This type of criteria has been analyzed by Hobson (2004) and the involved equivalent martingale measures are known as the q-optimal measures.

\section{Sensitivity ANALYSis FOR The OPtimal CONSUMPtion AND INVESTMENT POLICIES}

In this section, we revert our attention to the behavior of the optimal investment and consumption strategies with respect to the agent's preferences and the various market parameters. Specifically, we will investigate how conservative and aggressive agents react to changes in the levels of the stochastic factor, the correlation, the trading horizon and the $K_{i}$ utility coefficients.

These questions have been studied by various authors for special cases of model dynamics, preference choice and market incompleteness. We note that for most of the existing models (see, for example, Kim and Omberg (1996), Wachter (2002) and Campbell et. al. (2003)), the stochastic factor is modeled as the Sharpe ratio through the choice $\lambda(y, t)=y$ and $Y$ is taken to be a (possibly negative) OrnsteinUhlenbeck process. This assumption considerably simplifies the sensitivity analysis since it yields optimal portfolios that are linear in $Y$, but does not cover other interesting cases, such as models that include a predictability variable or allow for imperfectly correlated stochastic volatility. Moreover, such models have the awkward feature that an increase in $Y$ is sometimes perceived as an improvement in the stochastic opportunities (say for positive values of the Sharpe ratio) and sometimes as a worsening in the opportunity set (when the Sharpe ratio is so negative that shorting the stock becomes attractive). 
In our more general setting, we will see that the sign of $\lambda_{y} \lambda$ will play an important part in our understanding of the stochastic factor and will help us analyze the optimal investment and consumption behavior. If $\lambda_{y} \lambda>0$ for all possible values of the stochastic factor, we will refer to an increase in $y$ as an improvement in the investment opportunity set, while a decrease in $y$ will represent a worsening in the investment opportunity set. An example that obeys this assumption is the choice $\mu(y, t)=\mu_{0} y$ and $\sigma(y, t)=\sigma_{0} \sqrt{y}$, when the stochastic factor $Y$ is modeled as a positive process. In such a setting, an increase in $y$ is unambiguously an improvement in investment opportunities.

Conversely, if $\lambda_{y} \lambda<0$ for all possible values of the stochastic factor, we will refer to an increase in $y$ as a worsening in the investment opportunity set, while a decrease in $y$ will represent an improvement in the investment opportunity set. A common example of this case is a stochastic volatility model where $\mu(y, t)=\mu_{0}$, $\sigma(y, t)=\sqrt{y}$ and the stochastic factor $Y$ is a positive process representing the volatility. Clearly, an increase in the volatility is perceived by the investor as a worsening in the investment opportunity set.

Before discussing the impact of the model parameters on the agent's behavior, we recall (cf. Proposition 8) that the optimal consumption feedback rule is given by

$$
C^{*}(x, y, t)=K_{1}^{1 /(1-\gamma)} \frac{x}{h(y, t)}
$$

while the optimal portfolio $\pi_{s}^{*}$, consists of the myopic and non-myopic components,

$$
\pi^{*}(x, y, t)=\pi^{m}(x, y, t)+H(x, y, t)
$$

given by

$$
\pi^{m}(x, y, t)=\lambda(y, t) \frac{x}{\sigma(y, t)(1-\gamma)}
$$

and

$$
H(x, y, t)=\rho a(y, t) \frac{h_{y}(y, t)}{h(y, t)} \frac{x}{\sigma(y, t)}
$$

with $h$ solving (17)

\section{i) Sensitivity analysis}

As the next two Propositions illustrate, the sensitivity of the investment and consumption policies with respect to wealth $x$, time to maturity $T-t$, the correlation coefficient $\rho$ and the utility parameters $K_{1}$ and $K_{2}$ is the same for aggressive and conservative CRRA investors. However, the sensitivity of the policies with respect to changes in the level of the stochastic factor $y$, will depend crucially on the nature of the investor.

Proposition 12. For aggressive and conservative agents, the optimal consumption $C^{*}(x, y, t)$ has the following properties:

i) It is decreasing with respect to the ratio $K_{2} / K_{1}$ of utility parameters.

ii) It is linearly increasing in $x$. 
iii) If the coefficients $\lambda, b$ and $a$ are time homogeneous and

$$
-\left(\frac{K_{1}}{K_{2}}\right)^{1 /(1-\gamma)} \leq \frac{1}{2} \lambda^{2} \frac{\gamma}{(1-\gamma)^{2}},
$$

then it is decreasing with respect to the time to maturity, $T-t$.

iv) If the optimal investment is always positive, i.e. $\pi^{*}(x, y, t)>0$, and $\lambda_{y} \lambda>0$ (resp. $\left.\lambda_{y} \lambda<0\right)$ then it is decreasing (resp. increasing) in $\rho$.

Proposition 13. For aggressive and conservative investors:

i) The absolute value of the optimal portfolio $\left|\pi^{*}(x, y, t)\right|$ is linearly increasing in $x$.

ii) The optimal portfolio rule converges pointwise to the myopic investment as the trading horizon goes to zero, i.e.

$$
\lim _{t \rightarrow T} \pi^{*}(x, y, t)=\pi^{m}(x, y, t) .
$$

In the sequel, we will pay close attention to the properties that set conservative and aggressive agents apart. As it was discussed in Section 3, these two types of investors view the non-tradeable risk in the market very differently. This translates directly into different consumption and investment behaviors described, respectively, in Propositions 14 and 15. A striking feature of aggressive agents is that they consume a smaller proportion of their wealth when the investment opportunities improve. This behavior is known as the substitution effect (see Wachter (2002)), because as investment opportunities improve, they are willing to sacrifice some consumption today for higher expected consumption in the future. This is consistent with the fact that aggressive agents pursue investment strategies that pay off more than the myopic portfolio when the investment opportunities improve, a strategy we will refer to as speculating on improving investment opportunities. Whether this translates into a portfolio that is lower or higher than the myopic amount is simply an implementation issue that depends on the sign of the correlation $\rho$ and the sign of $\lambda \lambda_{y}$.

The investment and consumption behavior of conservative agents with respect to changes in the stochastic factor is drastically different. For example, conservative agents always yield to the so-called income effect: as the investment opportunities grow, they consume a larger proportion of their wealth. Moreover, they pursue investment strategies that pay off more than the myopic portfolio when the investment opportunities deteriorate. Such strategies will be referred to as hedging against worsening investment opportunities.

Proposition 14. The optimal consumption rule $C^{*}(x, y, t)$ has the following properties:

i) if the agent is aggressive, i.e. $\gamma \in(0,1)$, he/she consumes less when the investment opportunities improve (i.e. either $\lambda_{y} \lambda>0$ and $y$ increases, or $\lambda_{y} \lambda<0$ and $y$ decreases).

ii) if the agent is conservative, i.e. $\gamma \in(-\infty, 0)$, he/she consumes more when the investment opportunities improve (i.e. either $\lambda_{y} \lambda>0$ and $y$ increases, or $\lambda_{y} \lambda<0$ and $y$ decreases). 
Proposition 15. The non-myopic investment rule $H(x, y, t)$ has the following properties:

i) If the agent is aggressive, i.e. $\gamma \in(0,1)$, he/she speculates on improving investment opportunities. The implementation of this strategy is achieved by the following non-myopic policies:

$$
\begin{aligned}
& H(x, y, t)>0 \quad \text { if } \quad \lambda_{y} \lambda>0 \text { and } \rho>0, \quad \text { or if } \lambda_{y} \lambda<0 \text { and } \rho<0 \\
& H(x, y, t)<0 \text { if } \lambda_{y} \lambda>0 \text { and } \rho<0, \quad \text { or if } \lambda_{y} \lambda<0 \text { and } \rho>0
\end{aligned}
$$

ii) If the agent is conservative, i.e. $\gamma \in(-\infty, 0)$, he/she hedges against worsening investment opportunities. The implementation of this strategy is achieved by the following non-myopic policies:

$$
\begin{aligned}
& H(x, y, t)<0 \text { if } \lambda_{y} \lambda>0 \text { and } \rho>0 \text {, or if } \lambda_{y} \lambda<0 \text { and } \rho<0 \\
& H(x, y, t)>0 \text { if } \lambda_{y} \lambda>0 \text { and } \rho<0 \text {, or if } \lambda_{y} \lambda<0 \text { and } \rho>0
\end{aligned}
$$

Before turning to some numerical results, we present some natural bounds for the consumption rule.

Proposition 16. Let $C^{*}(x, y, t)$ be the optimal consumption rule, $M$ as in (25) and $\mathbb{P}^{\gamma}$ as in (20).

i) For aggressive agents,

$$
C^{*}(x, y, t) \geq K_{1}^{1 /(1-\gamma)} \frac{x}{\check{H}(y, t)}
$$

with $\check{H}$ given by $(34)$ and

$$
C^{*}(x, y, t) \leq \frac{x\left(\frac{K_{1}}{K_{2}}\right)^{1 /(1-\gamma)}}{\left(E_{\mathbb{P} \gamma}\left(M(t, T)^{1 / \delta} \mid Y_{t}=y\right)\right)^{\delta /(1-\gamma)}} .
$$

ii) For conservative agents,

$$
C^{*}(x, y, t) \leq \min \left(\frac{x K_{1}^{1 /(1-\gamma)}}{\check{H}(y, t)}, \frac{x\left(\frac{K_{1}}{K_{2}}\right)^{1 /(1-\gamma)}}{\left(E_{\mathbb{P}^{\gamma}}\left(M(t, T)^{1 / \delta} \mid Y_{t}=y\right)\right)^{\delta /(1-\gamma)}}\right) .
$$

Remark: The lower and upper bounds given by $\check{H}$ are attained in the complete markets limiting case (i.e. when $\rho= \pm 1$ ). As it was first pointed out in Wachter (2002), this limiting case provides an interesting analogy to fixed income pricing. Indeed, $\check{H}(y, t)$ may be interpreted as the present value of a bond with coupons $K_{1}^{1 /(1-\gamma)}$ and principal $K_{2}^{1 /(1-\gamma)}$.

\section{ii) Numerical Results}

In the remainder of this section, we will illustrate some of the results stated in the Propositions above, by deriving the optimal consumption and investment policies as a function of the Sharpe ratio, for various choices of model parameters, namely $\rho, \gamma, K_{2} / K_{1}$ and $T-t$. We will assume that the stock price and the stochastic factor solve

$$
d S_{s}=\left(0.08+\frac{0.14}{\pi} \tan ^{-1}\left(Y_{s}\right)\right) S_{s} d s+0.2 S_{s} d W_{s}^{1}
$$


and

$$
d Y_{s}=-0.1 Y_{s} d s+0.3 d W_{s}
$$

respectively. This choice ensures that the Sharpe ratio, given by

$$
\lambda(y)=0.4+\frac{0.7}{\pi} \tan ^{-1}(y)
$$

satisfies the condition $\lambda_{y} \lambda>0$ and that all the coefficients satisfy the assumptions stated in Section 2.

We use an explicit finite difference scheme to solve equation (17) numerically and compute the function $h(y, t)$. Since the nonlinearity of the equation is mild (the PDE is quasilinear), a simple central difference scheme for the first and second order spacial derivatives yields satisfactory result. The consumption to wealth ratio $C / x$ follows directly from (55), and the non-myopic portfolio to wealth ratio $H / x$ is obtained from (57), after numerically differentiating $h(y, t)$. 


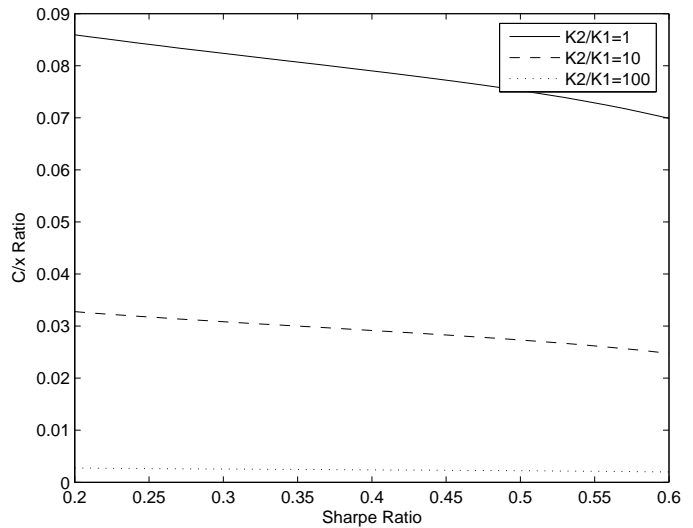

Figure 1. $\rho=-0.5, T-t=10$ and $\gamma=0.2$

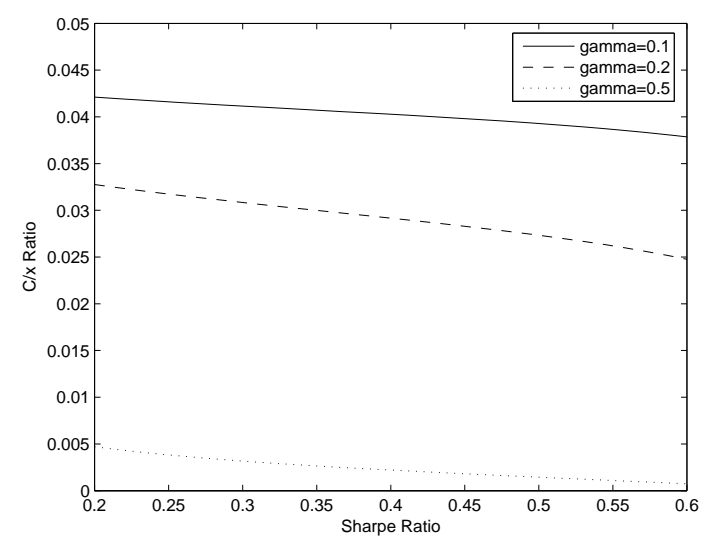

Figure 3. $\rho=-0.5, T=10$ and $K_{2} / K_{1}=10$

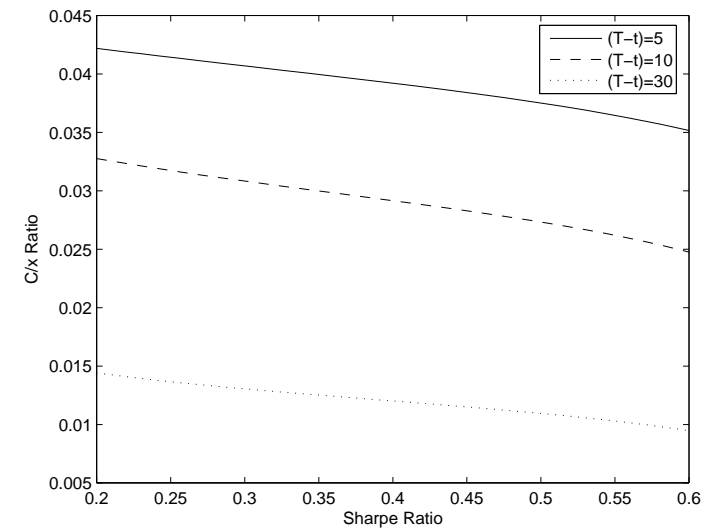

Figure 2. $\rho=-0.5, \gamma=0.2$ and $K_{2} / K_{1}=10$

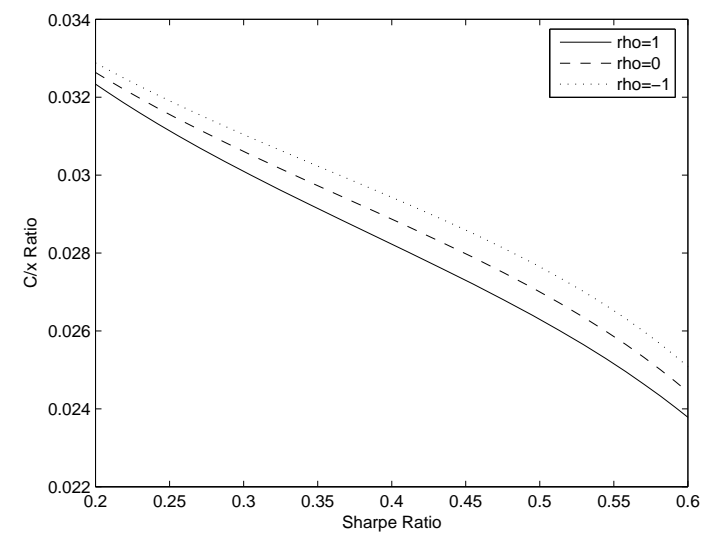

Figure $4 . K_{2} / K_{1}=10, T-t=10$ and $\gamma=0.2$

We first turn to the consumption behavior of aggressive agents, $\gamma \in(0,1)$. As it can be seen in the four figures above, the consumption to wealth ratio is decreasing in the Sharpe ratio. This illustrates the substitution effect as described in Proposition 14(i); as investment opportunities increase, due to a higher Sharpe ratio, the aggressive agents consume less since putting money aside is more powerful than consuming it right away.

Increasing the time horizon, $T-t$, or the relative importance of terminal wealth, $K_{2} / K_{1}$, both reduce the consumption to wealth ratio (see Figures 1 and 2), as predicted by Proposition 12. Note that the consumption is decreasing in $\rho$. As the parameter $\gamma$ increases, the agents seem to become "more aggressive" in the sense that they consume less (see Figure 3). 


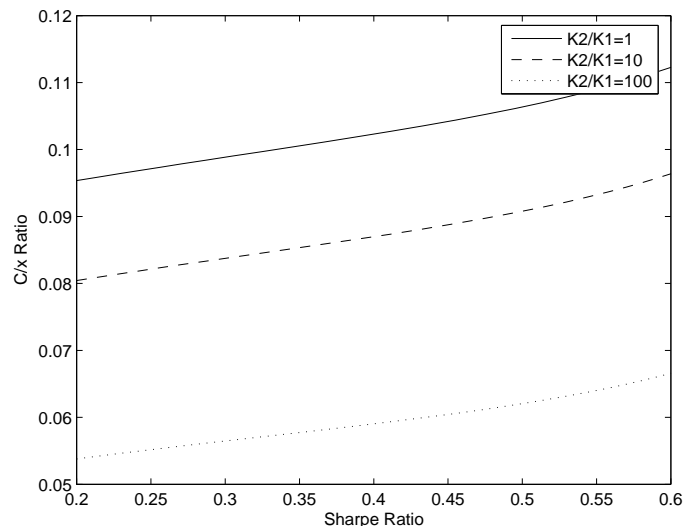

Figure 5. $\rho=-0.5, T-t=10$ and $\gamma=-1$

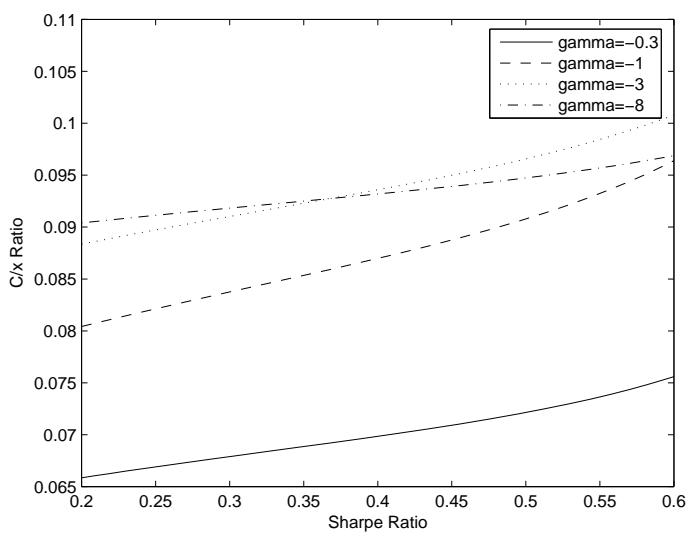

Figure 7. $\rho=-0.5, T-t=10$ and $K_{2} / K_{1}=10$

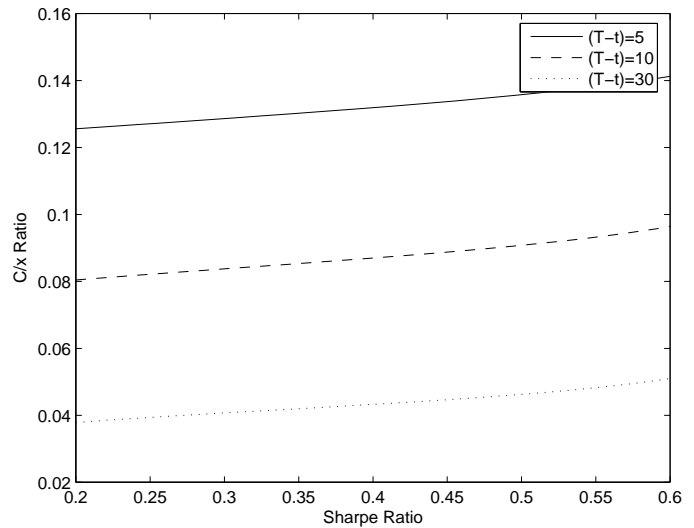

Figure 6. $\rho=-0.5, \gamma=-1$ and $K_{2} / K_{1}=10$

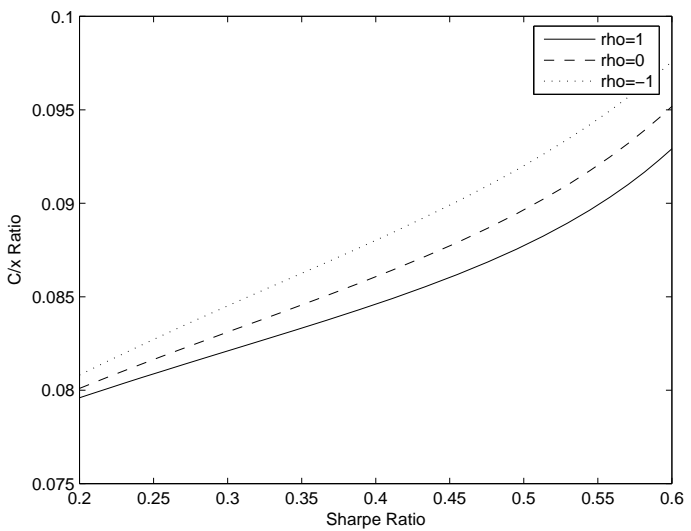

Figure $8 . K_{2} / K_{1}=10, T-t=10$ and $\gamma=-1$

We now turn to the consumption behavior of conservative agents, $\gamma<0$. In this case, we can observe the dominance of the income effect described in Proposition 14(ii). Indeed, as investment opportunities rise, due to a higher Sharpe ratio, the agent's consumption rises relative to current wealth.

Much like the case of aggressive agents, increasing the time horizon, $T-t$, or the relative importance of terminal wealth, $K_{2} / K_{1}$, both lead to a lower consumption policy (see Figures 5 and 6). Furthermore, the consumption is still decreasing in $\rho$. Figure 7 illustrates that the consumption policy is not monotonic in $\gamma$, so we cannot interpret this parameter as a measure of how "conservative" the agent is. 


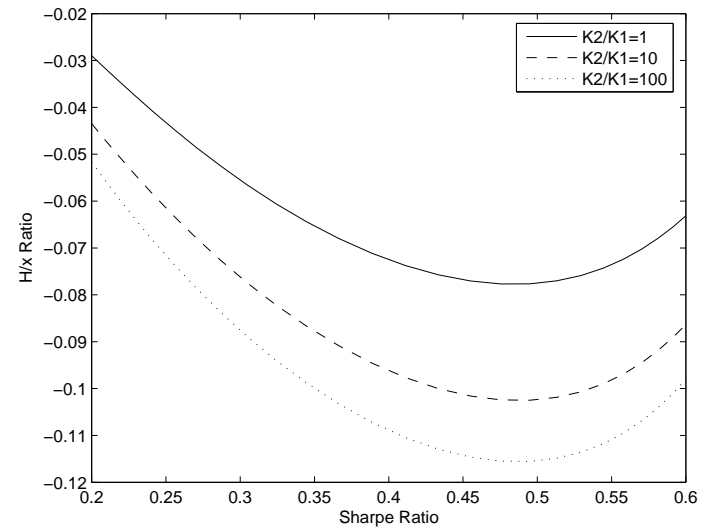

Figure 9. $\rho=-0.5, T-t=10$ and $\gamma=0.2$

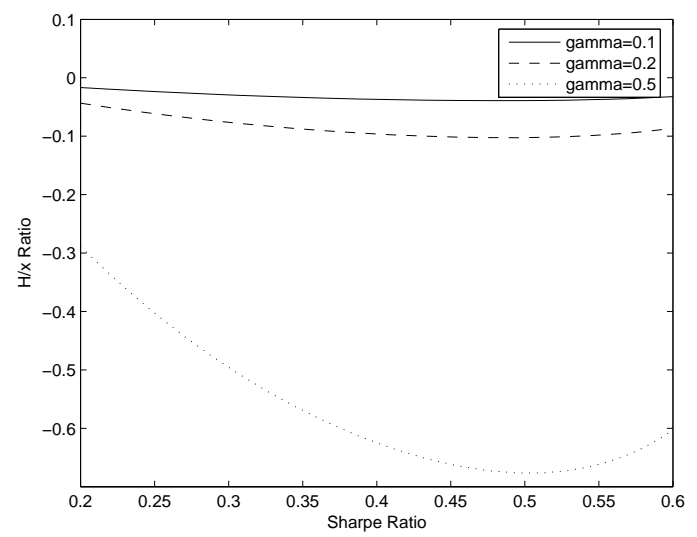

Figure 11. $\rho=-0.5, T-t=10$ and $K_{2} / K_{1}=10$

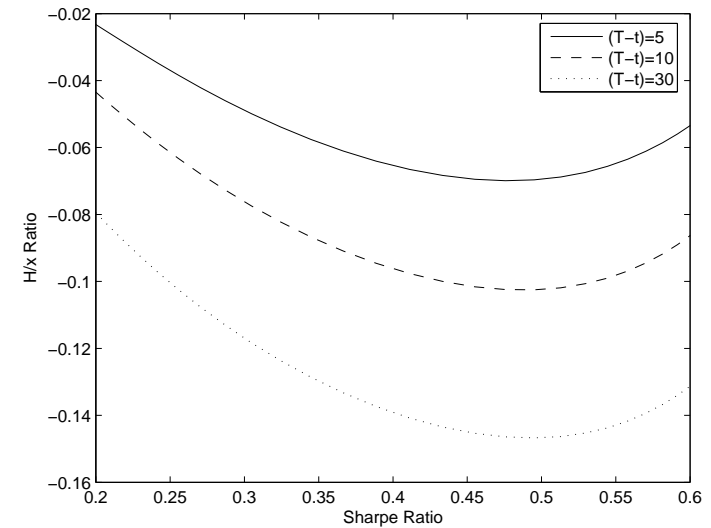

Figure 10. $\rho=-0.5, \gamma=0.2$ and $K_{2} / K_{1}=10$

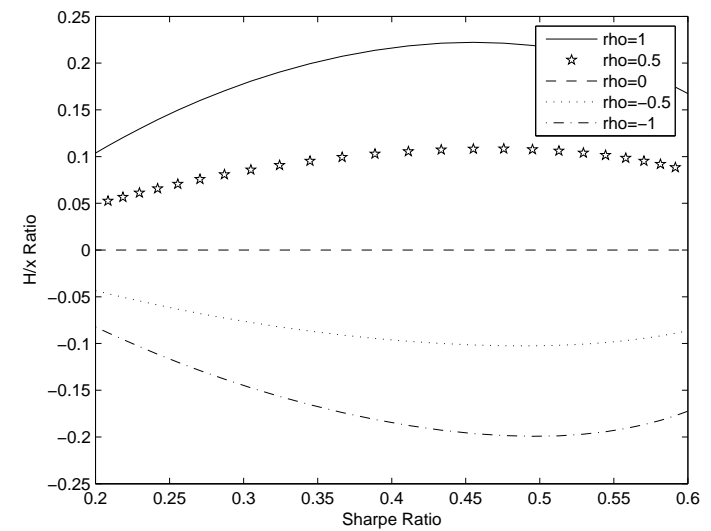

Figure $12 . K_{2} / K_{1}=10, T-t=10$ and $\gamma=0.2$

The next series of four graphs illustrates the non-myopic portfolio policy for aggressive agents, which is to short the stock if the correlation is negative and $\lambda \lambda_{y}>0$. Intuitively, since the Sharpe ratio tends to rise when the stock falls (since $\rho<0$ ), this shorting policy can be thought of as speculating on the Sharpe ratio. Notice that, unlike many of the existing models (see, for example, Kim and Omberg (1996) and Wachter (2002)) the ratio $H / x$ is not linear, or even increasing, in the Sharpe ratio. This is because our Sharpe ratio given by (60) is most variable around its central value of 0.4 and that is precisely when non-myopic hedging is most attractive.

As the time horizon, $T-t$, or the relative importance of the terminal wealth, $K_{2} / K_{1}$, increase, the agent's shorting policy increases in magnitude (see Figures 9 and 10). As $\gamma$ increases, the agent becomes more aggressive and the non-myopic portfolio can become dramatically large (see Figure 11). Finally notice that for positive values of $\rho$, the agent's behavior is reversed from being short in the stock to being long. 


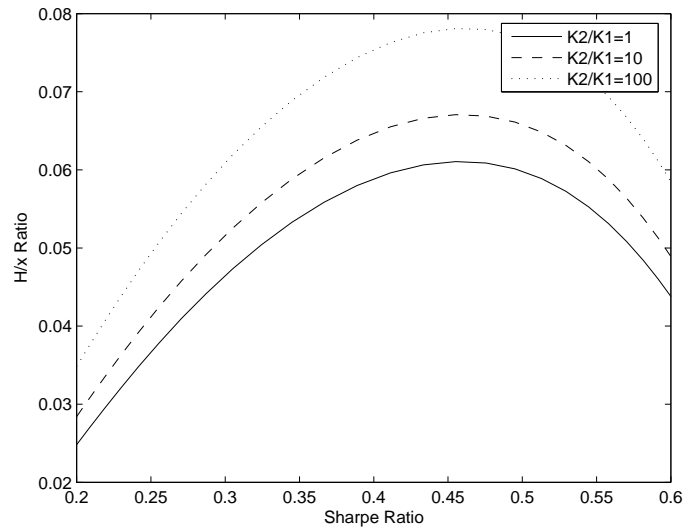

Figure 13. $\rho=-0.5, T-t=10$ and $\gamma=-1$

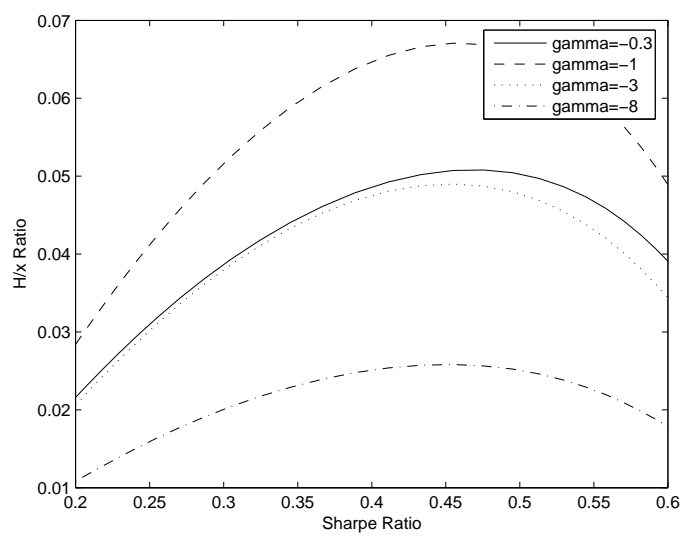

Figure 15. $\rho=-0.5, T-t=5$ and $K_{2} / K_{1}=10$

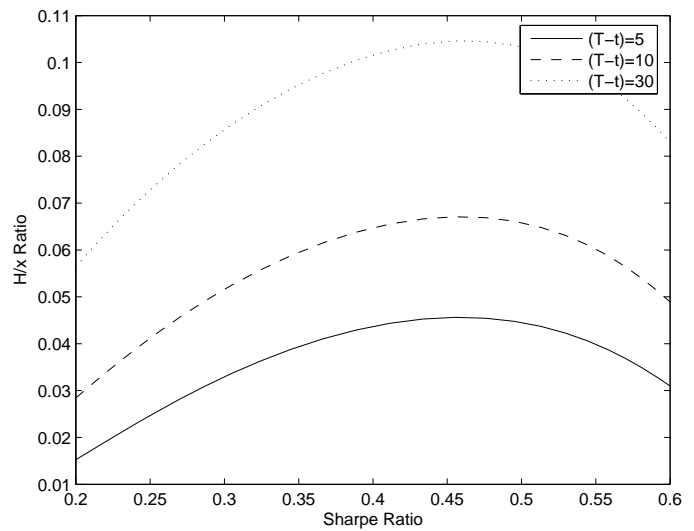

Figure 14. $\rho=-0.5, \gamma=-1$ and $K_{2} / K_{1}=10$

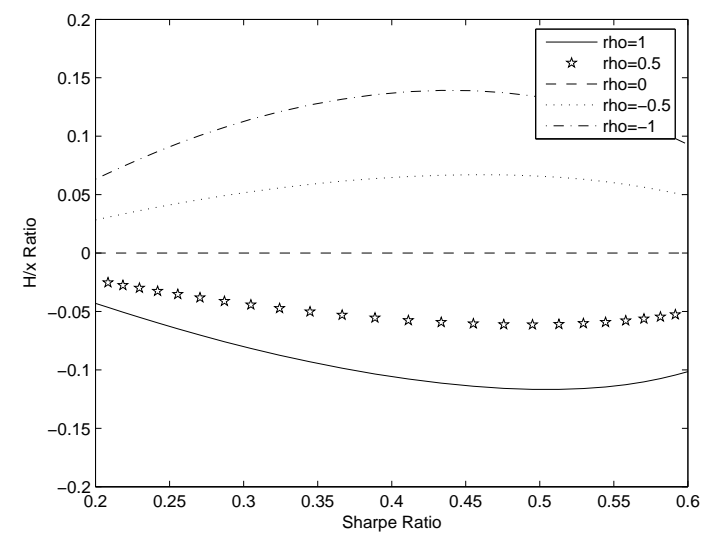

Figure 16. $K_{2} / K_{1}=10, T-t=10$ and $\gamma=-1$

The last four graphs illustrate that, if the correlation is negative and $\lambda \lambda_{y}>0$, the non-myopic portfolio policy for conservative agents is to be long in the stock. Since when the Sharpe ratio falls, the stock price tends to rise (due to $\rho<0$ ), this non-myopic policy can be interpreted as a hedge against worsening stochastic opportunities.

As the time horizon $T-t$ or the relative importance of the terminal wealth $K_{2} / K_{1}$ increase, the agent's non-myopic investment policy increases in magnitude (see Figures 13 and 14). Much like in the consumption policy for conservative agents, the non-myopic portfolio is not monotonic in $\gamma$ (see Figure 15). Finally notice that for positive values of $\rho$, the agent's behavior is reversed from being long in the stock to being short. 


\section{MARKET INCOMPLETENESS AND NON-ADDITIVE OPTIMAL BEHAVIOR}

In this section, we analyze the behavior of the value function and of the optimal policies in terms of the utility reward coming from intermediate consumption and from terminal wealth. In particular, we highlight an important qualitative difference between the complete and incomplete market setting. It is a well known fact that if the market is complete, the initial wealth $x$ can be split into two independent components, say $x^{w}$ and $x^{c}$ where $x^{w}+x^{c}=x$, each of which is invested optimally to solve a maximal expected utility sub-problem.

The first sub-problem is a pure optimal investment problem, financed by $x^{w}$, with utility coming from terminal wealth while the second is a pure consumption problem, financed by $x^{c}$, with utility coming solely from intermediate consumption. Intuitively, an investor who wants to invest money for a future date (say retirement) and consume some of his/her gains in the meantime, can effectively split the initial wealth into two accounts, one invested optimally to satisfy retirement needs, and one invested optimally to provide a steady income flow. This linear decomposition also occurs in the optimal policies of the original problem in that they turn out to be equal to the sum of the optimal policies associated with the two sub-problems. This linear decomposition result for the value function and the optimal policies can be proved directly by duality (see Karatzas et. al. (1987)).

In the sequel, we will investigate if such a decomposition result holds when the market becomes incomplete. As a first step, we derive the complete market result working directly with the primal problem (see Proposition 17). We will represent the complete market in terms of a model with nonlinear stock dynamics (i.e. with stock dynamics given by (26)). The incomplete market case is presented in Proposition 18.

We first introduce the pure investment and pure consumption expected utility problems given respectively by

$$
\tilde{V}^{w}(x, S, t)=\sup _{\mathcal{A}^{w}} E_{\mathbb{P}}\left(K_{2} \frac{\left(\tilde{X}_{T}^{w}\right)^{\gamma}}{\gamma} \mid \tilde{X}_{t}^{w}=x, \tilde{S}_{t}=S\right)
$$

and

$$
\tilde{V}^{c}(x, S, t)=\sup _{\mathcal{A}} E_{\mathbb{P}}\left(\int_{t}^{T} K_{1} \frac{\tilde{C}_{s}^{\gamma}}{\gamma} d s \mid \tilde{X}_{t}^{w}=x, \tilde{S}_{t}=S\right)
$$

where the wealth processes $\tilde{X}^{w}$ and $\tilde{X}^{c}$ solve

$$
d \tilde{X}_{s}^{w}=\mu\left(\tilde{S}_{s}, s\right) \tilde{\pi}_{s}^{w} d s+\sigma\left(\tilde{S}_{s}, s\right) \tilde{\pi}_{s}^{w} d \tilde{W}_{s}
$$

and

$$
d \tilde{X}_{s}^{c}=\mu\left(\tilde{S}_{s}, s\right) \tilde{\pi}_{s}^{c} d s-C_{s} d s+\sigma\left(\tilde{S}_{s}, s\right) \tilde{\pi}_{s}^{c} d \tilde{W}_{s}
$$

The above two value functions can be seen as special cases of (27), which we derived in Section 2(iii). Using the results therein, we deduce that the two maximal expected utilities can be represented as

$$
\tilde{V}^{w}(x, S, t)=\frac{x^{\gamma}}{\gamma} \tilde{h}^{w}(S, t)^{1-\gamma}
$$


and

$$
\tilde{V}^{c}(x, S, t)=\frac{x^{\gamma}}{\gamma} \tilde{h}^{c}(S, t)^{1-\gamma}
$$

where $\tilde{h}^{w}$ and $\tilde{h}^{c}$ solve, respectively,

$$
\tilde{h}_{t}^{w}+\frac{1}{2} \sigma^{2} S^{2} \tilde{h}_{S S}^{w}+\frac{\mu}{1-\gamma} S \tilde{h}_{S}^{w}+\frac{\gamma}{2(1-\gamma)^{2}} \lambda^{2} \tilde{h}^{w}=0
$$

with $\tilde{h}^{w}(S, T)=K_{2}^{1 /(1-\gamma)}$, and

$$
\tilde{h}_{t}^{c}+\frac{1}{2} \sigma^{2} S^{2} \tilde{h}_{S S}^{c}+\frac{\mu}{1-\gamma} S \tilde{h}_{S}^{c}+\frac{\gamma}{2(1-\gamma)^{2}} \lambda^{2} \tilde{h}^{c}+K_{1}^{1 /(1-\gamma)}=0
$$

with $\tilde{h}^{c}(S, T)=0$.

Proposition 17. The value function $\tilde{V}$ of the optimal investment-consumption problem (27) is given by

$$
\tilde{V}(x, S, t)=\tilde{V}^{c}\left(x^{c}, S, t\right)+\tilde{V}^{w}\left(x^{w}, S, t\right)
$$

where

$$
x^{c}=x \frac{\tilde{h}^{c}}{\tilde{h}} \quad \text { and } \quad x^{w}=x\left(1-\frac{\tilde{h}^{c}}{\tilde{h}}\right) .
$$

Moreover, the optimal investment and consumption policies, $\tilde{\pi}_{s}^{*}$ and $\tilde{C}_{s}^{*}$, satisfy

$$
\tilde{\pi}_{s}{ }^{*}=\tilde{\pi}_{s}{ }^{w, *}+\tilde{\pi}_{s}^{c, *}
$$

and

$$
\tilde{C}_{s}^{*}=\tilde{C}_{s}^{c, *}
$$

where $\tilde{\pi}_{s}{ }^{w, *}$ is the optimal policy associated with the pure investment problem $\tilde{V}^{w}\left(x^{w}, S, t\right)$ and $\tilde{\pi}_{s}^{c, *}$ and $\tilde{C}_{s}^{c, *}$ are the optimal policies associated with the pure consumption problem $\tilde{V}^{c}\left(x^{c}, S, t\right)$.

The proof is provided in Appendix D and is based on the linearity of the involved equations (cf. (61) and (62)).

We now revert our attention to the incomplete market case and introduce, in analogy to the previous decomposition, the value functions,

$$
V^{w}(x, y, t)=\sup _{\mathcal{A}^{w}} E_{\mathbb{P}}\left(K_{2} \frac{\left(X_{T}^{w}\right)^{\gamma}}{\gamma} \mid X_{t}^{w}=x, Y_{t}=y\right)
$$

and

$$
V^{c}(x, y, t)=\sup _{\mathcal{A}} E_{\mathbb{P}}\left(\int_{t}^{T} K_{1} \frac{C_{s}^{\gamma}}{\gamma} d s \mid X_{t}^{w}=x, Y_{t}=y\right) .
$$

In incomplete markets, as the following Proposition illustrates, the separation result of Proposition 17 would not be optimal. In other words, if, at time $t$, the agent splits his/her wealth into two separate accounts, say $k x$ and $(1-k) x$, as soon as the investment opportunities changed, he/she would want to transfer money across the accounts. 
Proposition 18. Let $V$ be the value function of the optimal investment-consumption problem (7) and $V^{c}$ and $V^{w}$ be, respectively, the value functions of the pure consumption and investment problems. Then, for any $k=k(x, y, t)$,

$$
V(x, y, t) \geq V^{c}(x k, y, t)+V^{w}(x(1-k), y, t)
$$

where equality can be attained for some $k$ if and only if $\rho= \pm 1$ or $a=0$.

The proof is provided in Appendix D.

\section{BiBLIOGRAPHY}

Barles, G. (1994): Viscosity solutions of Hamilton-Jacobi equations, Mathematics and Applications, 17, Springer-Verlag, Paris.

Bellini, F. and M. Fritelli (2002): On the existence of minimax martingale measures, Mathematical Finance, 12, 1-21.

Blake, D. (1996): Efficiency, risk aversion and portfolio insurance: an analysis of financial asset portfolios held by investors in the United Kingdom, Economic Journal, 106, 1175-92.

Bouchard, B. (2002): Stochastic target with mixed diffusion processes, Stochastic Processes and their Applications, 101, 273-302.

Bouchard, I. Ekeland and N. Touzi (2004): On the Malliavin approach to Monte Carlo approximation of conditional expectations, Finance and Stochastics, 8, 45-71.

Campbell, J.Y. and L.M. Viceira (1999): Consumption and portfolio decisions when expected returns are time-varying, Quarterly Journal of Economics, 114, 433495 .

Campbell, J.Y., Chacko, G., Rodriguez, J. and L.M. Viceira (2003): Strategic asset allocation in a continuous-time VAR model, Journal of Economic Dynamics and Control, forthcoming.

Campbell, J. Y., Cocco, J., Gomes, F., Maenhout, P. J. and L.M. Viceira (2004): Stock market mean reversion and the optimal equity allocation of a long-lived investor, European Finance Review, in press.

Capuzzo-Dolcetta, I., and P.-L. Lions (1990): Viscosity solutions of HamiltonJacobi Equations, Transactions Amer. Math. Soc., 318, 543-683.

Chacko, G. and L. Viceira. (1999): Dynamic consumption and portfolio choice with stochastic volatility in incomplete markets, NBER Working papers, No 7377.

Cont, R., Tankov, P. and E. Voltchkova (2004): Option pricing models with jumps: integro-differential equations and inverse problems. P. Naittaanmki, T. Rossi, S. Korotov, E. Onate, J. Priaux and D. Knorzer (eds.) European Congress on Computational Methods in Applied Sciences, Jyvskyl. 
Cox, J.C. and C.F. Huang (1989): Optimal consumption and portfolio policies when asset prices follow a diffusion process, J. Economic Theory, 49, 33-83.

Crandall, M.G., Ishii, H. and P.-L. Lions (1992): User's guide to viscosity solutions of second order partial differential equations, Bull. Amer. Math. Soc. (N.S.) $27,1-67$.

Crandall, M.G. and P.-L. Lions (1983): Viscosity solutions of Hamilton-Jacobi equations, Transactions Ame. Math. Soc., 277, 1-42.

Cvitanić, J. and I. Karatzas (1992): Convex duality in constrained portfolio optimization, Ann. Appl. Probab. 2, 767-818.

Davis, M.H.A. (2000): Optimal hedging with basis risk, preprint.

Davis, M.H.A., Panas, V.G., and T. Zariphopoulou (1993): European option pricing with transaction costs. SIAM J. Control Optim. 31, 470-493.

Delbaen, F., Grandits, P., Rheinlander, T., Samperi, D., Schweizer, M. and C. Stricker (2002): Exponential hedging and entropic penalties, Mathematical Finance, 12, 99-123.

Duffie D., Fleming W.H., Soner H.M. and T. Zariphopoulou (1997): Hedging in incomplete markets with HARA utility, Journal of Economic Dynamics and Control, 21, 753-782.

Duffie, D. and T. Zariphopoulou (1993): Optimal investment with undiversifiable income risk. Mathematical Finance, 3, 135-148.

Dybvig, P. and C.-F. Huang (1988): Nonnegative wealth, absence of arbitrage, and feasible consumption plans, Review of Financial Studies, 1, 377-401.

El Karoui, N. and M.C. Quenez (1997): Non-linear pricing theory and backward stochastic differential equations. Financial mathematics (Bressanone, 1996), Lecture Notes in Math., 1656, Springer, Berlin, 191-246.

Fitzpatrick, B. and W. Fleming (1990): Numerical methods for optimal investment - Consumption models. in Proc. 29th IEEE Conf. Decision and Control.

Fleming, W.H. and H.M. Soner (1993): Controlled Markov processes and viscosity solutions. Berlin Heidelberg New York: Springer.

Fleming, W.H. and T. Zariphopoulou (1991): An optimal investment/consumption model with borrowing. Math. Oper. Res. 16, 802-822.

He, H., and N.D. Pearson (1991): Consumption and portfolio policies with incomplete markets and short-sale constraints: the infinite-dimensional case, $J$. Econom. Theory, 54, no. 2, 259-304.

Henderson, V. and D. Hobson (2002): Real options with constant relative risk aversion, Journal of Economic Dynamics and Control, 27, 329-355. 
Hobson, D. (2004): Stochastic volatility models, correlation and the q-optimal measure, Mathematical Finance, 14, 537-556.

Ishii, H. and P.-L. Lions (1990): Viscosity solutions of fully nonlinear secondorder elliptic partial differential equations, J. Differential Equations, 83, 26-78.

Jacobs, K. (2002): The rate of risk aversion may be lower than you think, working paper.

Kabanov, Y. and C. Stricker (2002): On the optimal portfolio for the exponential utility maximization, remarks to the six-author paper "Exponential hedging and entropic penalties", Mathematical Finance, 12, 125-134.

Kaplow, L. (2003): The value of a statistical life and the coefficient of relative risk aversions, NBER working paper.

Karatzas, I., Lehoczky, J.P. and S.E. Shreve (1987): Optimal portfolio and consumption decisions for a small investor on a finite horizon, SIAM Journal of Control and Optimization, 25, 1557-1586.

Karatzas, I. and G. Zitkovic (2003): Optimal consumptions from investment and random endowment in incomplete semimartingale markets", Annals of Probability, $31,1821-1858$.

Katsoulakis, M.A. (1994): Viscosity solutions of second order fully nonlinear elliptic equations with state constraints, Indiana University Mathematical Journal, 43, 493-518.

Kim, T.S. and E. Omberg (1996): Dynamic non-myopic portfolio behavior, Review of Financial Studies, 9, 141-161.

Korn, R. and H. Kraft (2004): On the stability of continuous-time portfolio problems with stochastic opportunity set, Mathematical Finance, 14, 403-414.

Kraft, H. (2003): Optimal portfolios and Heston's stochastic volatility model, working paper.

Kramkov, D. and W. Schachermayer (1999): The asymptotic elasticity of utility functions and optimal investments in incomplete markets, Annals of Applied Probability, 9, 904-950.

Krylov, N.V. (1996): Introduction to the theory of diffusion processes, AMS, Providence, (translation of Russian original, 1989).

Lions, P.-L. (1983): Optimal control of diffusion processes and Hamilton-JacobiBellman equations. Part I: The dynamic programming principle and applications. Part II: Viscosity solutions and uniqueness. Comm. Partial Differential Equations, 8, 1101-1174 and 1229-1276.

Liu, J. (1999): Portfolio selection in stochastic environments, working paper, Stanford University. 
Mehra, R., and E.C. Prescott (1985): The equity premium: a puzzle. Journal of Monetary Economics, 15, 145-161.

Merton, R.C. (1969): Lifetime portfolio selection under uncertainty: the continuous time case, Review of Economics and Statistics, 51, 247-257.

Merton, R. C. (1971): Optimum consumption and portfolio rules in a continuoustime model, J. Econom. Theory, 3, 373-413.

Monoyios, M. (2004): Characterization of optimal dual measures via distortion, working paper.

Palsson, A-M. (1996): Does the degree of relative risk aversion vary with household characteristics? Journal of Economic Psychology, 17, 771-787.

Pang, T. (2004): Portfolio optimization models on infinite-time horizon. Journal of optimization theory and applications, 122, 573-597.

Pham, H. (2002): Smooth solutions to optimal investment models with stochastic volatilities and portfolio constraints, Applied Mathematics and Optimization, 46, $55-78$.

Pliska, S.R. (1986): A stochastic calculus model of continuous trading: optimal portfolios, Mathematics of Operations Research, 11, 239-246.

Protter, M and H. Weinberger (1967): Maximum principles in differential equations, Prentice-Hall.

Rogers, L.C.G. (2002): Duality in constrained optimal investment and consumption problems: a synthesis. Paris-Princeton Lectures on Mathematical Finance, (Springer Lecture Notes in Mathematics 1814), 95-131.

Rothe, F. (1984): Global solutions of reaction-diffusion systems, Lecture Notes in Mathematics, 1072. Springer-Verlag, Berlin.

Rouge, R. and El Karoui, N. (2000): Pricing via utility maximization and entropy, Mathematical Finance, 10, 259-276.

Schroder, M. and C. Skiadas (2003): Optimal lifetime consumption-portfolio strategies under trading constraints and generalized recursive preferences, Stochastic Processes and their Applications, 108, 155-202.

Shreve, S. E. and H.M. Soner (1994): Optimal investment and consumption with transaction costs, Ann. Appl. Probab., 4, 609-692.

Schachermayer, W. (2001): Optimal investment in incomplete markets when wealth may become negative, Annals of Applied Probability, 11, 694-734.

Smoller, J. (1994): Shock waves and reaction-diffusion equations. Second edition. Grundlehren der Mathematischen Wissenschaften [Fundamental Principles of Mathematical Sciences], 258. Springer-Verlag, New York. 
Soner, H. M. (1986): Optimal control with state-space constraint, SIAM J. Control Optim., 24, 1110-1122.

Soner, H. M. (1997): Controlled Markov processes, viscosity solutions and applications to mathematical finance. Viscosity solutions and applications (Montecatini Terme, 1995), 134-185, Lecture Notes in Math., 1660, Springer, Berlin.

Tehranchi, M. (2004): Explicit solutions of some utility maximization problems in incomplete markets, Stochastic Processes and Their Applications, 114, 109-125.

Tiu, C. (2002): On the Merton Problem in Incomplete Markets. The University of Texas at Austin, Ph.D. Thesis.

Tiu, C. (2004): A new technique for hedging under partial information, working Paper.

Tourin A., and T. Zariphopoulou (1994): Numerical schemes for investment models with singular transactions. Computational Economics, 7, 287-307.

Touzi, N. (2004): Stochastic control problems, viscosity solutions and application to finance. Publications of the Scuola Normale Superiore of Pisa.

Wachter, J. (2002): Portfolio consumption decisions under mean-reverting returns: an exact solution for complete markets, Journal of Financial and Quantitative Analysis, 37, 63-91.

Zariphopoulou, T. (1988): Optimal investment-consumption models with constraints, Brown University, Ph.D. Thesis.

Zariphopoulou, T. (1992): Investment-consumption models with transaction fees and Markov-chain parameters. SIAM J. Control Optim., 30, 613-636.

Zariphopoulou, T. (1999): Optimal investment and consumption models with nonlinear stock dynamics, Mathematical Methods of Operations Research, 50, 271296.

Zariphopoulou, T. (2001): A solution approach to valuation with unhedgeable risks, Finance and Stochastics, 5, 61-82.

Zariphopoulou, T. (2003): Stochastic control methods in asset pricing, Handbook of Stochastic Analysis and Applications, D. Kannan and V. Lakshmikantham (eds), Marcel Dekker, 102-145. 


\section{Appendices}

\section{Appendix A.}

We start with the definition of constrained viscosity solutions (see Soner (1986) and Capuzzo-Dolcetta and Lions (1987) for first order equations, and Lions (1983), Ishii and Lions (1990), Katsoulakis (1994) for second order ones).

Consider a fully nonlinear second order partial differential equation of the form

$$
F\left(z, t, u, u_{t}, \nabla u, \nabla^{2} u\right)=0 \quad \text { in } \quad G
$$

where $G=\mathcal{R} \times \mathcal{R} \times[0, T]$, and $F: \mathcal{R}^{2} \times[0, T] \times \mathcal{R} \times \mathcal{R}^{2} \times \mathcal{R}^{4} \rightarrow \mathcal{R}$ is continuous and (degenerate) elliptic, meaning that $F(z, t, p, q, X, A+B) \leq F(z, t, p, q, X, A)$ if $B>0$.

Definition 19. A continuous function $u: \bar{G} \rightarrow \mathcal{R}$ is a constrained viscosity solution of (65) on $\bar{G}$ if

i) $u$ is a viscosity subsolution of (65) on $\bar{G}$, that is, for any $\phi \in C^{2,1}(\bar{G})$ and any local maximum point $\left(z_{0}, t_{0}\right) \in \bar{G}$ of $u-\phi$,

$$
F\left(z_{0}, t_{0}, u\left(z_{0}, t_{0}\right), \phi_{t}\left(z_{0}, t_{0}\right), \nabla \phi\left(z_{0}, t_{0}\right), \nabla^{2} \phi\left(z_{0}, t_{0}\right)\right) \leq 0,
$$

and

ii) $u$ is a viscosity supersolution of (65) in $\bar{G}$, that is, for any $\phi \in C^{2,1}(\bar{G})$ and any local minimum point $\left(z_{0}, t_{0}\right) \in \bar{G}$ of $u-\phi$,

$$
F\left(z_{0}, t_{0}, u\left(z_{0}, t_{0}\right), \phi_{t}\left(z_{0}, t_{0}\right), \nabla \phi\left(z_{0}, t_{0}\right), \nabla^{2} \phi\left(z_{0}, t_{0}\right)\right) \geq 0,
$$

where $\nabla \phi$ and $\nabla^{2} \phi$ denote, respectively, the gradient vector and the second derivative matrix of $\phi$.

To establish the results of Proposition 1, we follow the arguments used in the proofs of Theorem 4.1 and Theorem 4.2 in Duffie and Zariphopoulou (1993), appropriately extended to accommodate the time-dependent character of the problem. We need to show that $V$ is a constrained viscosity solution to the HJB equation and that it is the unique such solution in the appropriate class. We start with the former question.

Generally speaking, to establish the constrained viscosity property of the value function one can follow the arguments of Ishii and Lions (1990). However, their arguments require that the set of admissible policies is compact, a fact that is violated for the admissible portfolios and consumptions plans $(\pi, C) \in \mathcal{A}$. This situation is remedied by first considering an approximate stochastic control problem with admissible controls $\left(\pi, C^{N}\right) \in \mathcal{A}_{N}$ with $C_{s}^{N}=\min \left(C_{s}, N\right)$ for $t \leq s \leq T$ and $N>0$. We denote the value function of this problem by $V^{N}$ and we work with the so-called "normalized" version of the model. ${ }^{1}$

To this end, we introduce an appropriate random change of time and we work with the state dynamics and the value function of the normalized model. Following

\footnotetext{
${ }^{1}$ The latter method, introduced by Krylov (1996) (see also Lions (1983)) was first applied in optimal portfolio choice problems by Duffie and Zariphopoulou (1993).
} 
classical arguments of stochastic control, we then establish that the value function $V^{N}$ is a constrained viscosity solution of the normalized HJB equation, namely,

$$
\begin{gathered}
\max _{\pi} \frac{1}{1+\pi^{2}}\left(V_{t}^{N}+\left(\frac{1}{2} \sigma^{2} \pi^{2} V_{x x}^{N}+\pi\left(\rho \sigma a V_{x y}^{N}+\mu V_{x}^{N}\right)\right)\right. \\
\left.+\max _{c \leq N}\left(-c V_{x}^{N}+K_{1} \frac{c^{\gamma}}{\gamma}\right)+\mathcal{L} V^{N}\right)=0 .
\end{gathered}
$$

Working as in the first part of the proof in Theorem 4.1 in Duffie and Zariphopoulou (Cases $A$ to $E$ ) we show that $V^{N}$ is actually a constrained viscosity solution of the original HJB equation. To complete the first part of the proof, we need to establish -using the stability properties of viscosity solutions (see, Lions (1984))- that as $N \rightarrow \infty, V^{N} \rightarrow V$, locally uniformly on $\bar{D}$. But this follows from the monotonicity properties of the utility function and the value function, as well as the monotone convergence theorem. We note that the time-dependent feature of the problem does not pose any difficulty for the validity of the arguments used to establish the viscosity properties of $V$.

To show that $V$ is the unique constrained viscosity solution, we need to modify the arguments of Theorem 4.2 in Duffie and Zariphopoulou (1993) in order to accommodate the time-dependence. To this end, we denote with a slight abuse of notation, by $u$ and $v$, viscosity sub- and super-solutions of (10) that are concave (of $\gamma$-sublinear growth, similar to the growth of the utility functionals), increasing in $x$ and for fixed $y$, they are bounded uniformly in $(x, t)$. We next assume that $u(x, y, T) \leq v(x, y, T)$ and that $u(0, y, T)=v(0, y, T)$. We then consider an arbitrary constant $m>0$, define the function $\Phi^{m}(x, y, t)=u(x, y, t)-v(x, y, t)-m(T-$ $t)$ and look at $\sup _{(x, y, t) \in \overline{\mathcal{D}}} \Phi^{m}(x, y, t)$. Clearly, if $\sup _{(x, y, t) \in \overline{\mathcal{D}}} \Phi^{m}(x, y, t)=0$ for all $m>0$, the comparison follows from using that $u(x, y, T)=v(x, y, T)$ and passing to the limit as $m \downarrow 0$. It remains to investigate if comparison holds for the other case, i.e. in the case that there exists $\bar{m}>0$ with $\sup _{(x, y, t) \in \overline{\mathcal{D}}} \Phi^{\bar{m}}(x, y, t)>0$ and the maximum occurs at a point $\left(x_{0}, y_{0}, t_{0}\right) \in \overline{\mathcal{D}}$ with $t_{0}<T$. We are going to establish that this case cannot occur. For this, we consider the function $\tilde{u}=u-\bar{m}(T-t)$ and we define for $z=(x, y), \bar{z}=(\bar{x}, \bar{y})$ for $x, \bar{x} \in \mathcal{R}^{+}$and $y, \bar{y} \in \mathcal{R}$ the function

$$
\phi(z, \bar{z}, t)=\left|\frac{z-\bar{z}}{\delta}-8 \eta\right|^{4}+\theta e^{-\zeta(x+y)}+\bar{m}(T-t)
$$

where $\eta \in \mathcal{R}^{2}$, and appropriately chosen constants $\zeta \in(\gamma, \infty), \theta$ and $\delta$.

From the growth assumptions on $u$ and $v$, the definition of $\tilde{u}$ and the role of $\bar{m}$, we get that the maximum of $\psi(z, \bar{z}, t)=u(z, \bar{z}, t)-v(z, \bar{z}, t)-\phi(z, \bar{z}, t)$ occurs at a point, say $\left(z^{*}, \bar{z}^{*}, t^{*}\right)$, that converges to $\left(x_{0}, y_{0}, t_{0}\right)$ as $\delta, \theta \downarrow 0$.Using a straightforward variation of the arguments used in Theorem 4.2 of Duffie and Zariphopoulou (1993), we get, after tedious but routine calculations, that $\bar{m} \leq 0$, which is a contradiction.

Proof of Proposition \%: To facilitate the proof, we first establish a uniform lower bound for the function $h$. We recall that $h$ solves

$$
h_{t}+\frac{1}{2} a^{2} h_{y y}+\left(b+\rho \frac{\gamma}{1-\gamma} \lambda a\right) h_{y}+\frac{1}{2} \frac{\gamma}{(1-\gamma)^{2}} \lambda^{2} h-\frac{1}{2} \gamma\left(1-\rho^{2}\right) a^{2} \frac{h_{y}^{2}}{h}+K_{1}^{1 /(1-\gamma)}=0
$$


with $h(y, T)=K_{2}^{1 /(1-\gamma)}$. Since $K_{1}>0$,

$$
h_{t}+\frac{1}{2} a^{2} h_{y y}+\left(b+\rho \frac{\gamma}{1-\gamma} \lambda a\right) h_{y}+\frac{1}{2} \frac{\gamma}{(1-\gamma)^{2}} \lambda^{2} h-\frac{1}{2} \gamma\left(1-\rho^{2}\right) a^{2} \frac{h_{y}^{2}}{h} \leq 0
$$

which in turn yields that $h$ is a supersolution to

$$
\hat{H}_{t}+\frac{1}{2} a^{2} \hat{H}_{y y}+\left(b+\rho \frac{\gamma}{1-\gamma} \lambda a\right) \hat{H}_{y}+\frac{1}{2} \frac{\gamma}{(1-\gamma)^{2}} \lambda^{2} \hat{H}-\frac{1}{2} \gamma\left(1-\rho^{2}\right) a^{2} \frac{\hat{H}_{y}^{2}}{\hat{H}}=0
$$

with $\hat{H}(y, T)=K_{2}^{1 /(1-\gamma)}$. Direct calculations (see, Zariphopoulou (2001) Theorem 3.2 ) yield that

$$
\hat{H}(y, t)=v^{w}(y, t)^{\delta /(1-\gamma)}=K_{2}^{1 /(1-\gamma)}\left(E_{\mathbb{P} \gamma}\left(M(t, T)^{1 / \delta} \mid Y_{t}=y\right)\right)^{\delta /(1-\gamma)} .
$$

The supersolution property of $h$ together with classical comparison results for quasilinear equations (see, for example, Theorem 3.12 in Protter and Weinberger (1967)), gives that

or, equivalently, that

$$
h(y, t) \geq \hat{H}(y, t)
$$

$$
h(y, t)^{1-\gamma} \geq K_{2}\left(E_{\mathbb{P} \gamma}\left(M(t, T)^{1 / \delta} \mid Y_{t}=y\right)\right)^{\delta} .
$$

From Proposition 3 , we then see that, if $\gamma \in(0,1)$, multiplying the above inequality by $x^{\gamma} / \gamma$ yields

$$
\frac{x^{\gamma}}{\gamma} h(y, t)^{1-\gamma} \geq K_{2} \frac{x^{\gamma}}{\gamma}\left(E_{\mathbb{P} \gamma}\left(M(t, T)^{1 / \delta} \mid Y_{t}=y\right)\right)^{\delta}
$$

and (32) follows. The inequality is reversed if $\gamma \in(-\infty, 0)$, yielding (35).

We continue with the upper bound (33) for the value function of the aggressive agent. Considering again the equation (17) we see that

$$
\begin{gathered}
0=h_{t}+\frac{1}{2} a^{2} h_{y y}+\left(b+\rho \frac{\gamma}{1-\gamma} \lambda a\right) h_{y}+\frac{1}{2} \frac{\gamma}{(1-\gamma)^{2}} \lambda^{2} h-\frac{1}{2} \gamma\left(1-\rho^{2}\right) a^{2} \frac{h_{y}^{2}}{h}+K_{1}^{1 /(1-\gamma)} \\
\leq h_{t}+\frac{1}{2} a^{2} h_{y y}+\left(b+\rho \frac{\gamma}{1-\gamma} \lambda a\right) h_{y}+\frac{1}{2} \frac{\gamma}{(1-\gamma)^{2}} \lambda^{2} h+K_{1}^{1 /(1-\gamma)}
\end{gathered}
$$

where we used that $\gamma \in(0,1)$ and $h>0$. Therefore, $h$ is a subsolution of

$$
\check{H}_{t}+\frac{1}{2} a^{2} \check{H}_{y y}+\left(b+\rho \frac{\gamma}{1-\gamma} \lambda a\right) \check{H}_{y}+\frac{1}{2} \frac{\gamma}{(1-\gamma)^{2}} \lambda^{2} \check{H}+K_{1}^{1 /(1-\gamma)}=0
$$

with $\check{H}(y, T)=K_{2}^{1 /(1-\gamma)}$ and therefore,

$$
h(y, t) \leq \check{H}(y, t) .
$$

Direct application of the Feynman-Kac formula yields the probabilistic representation

$$
\check{H}(y, t)=E_{\mathbb{P} \gamma}\left(\int_{t}^{T} M(t, s)^{1 /(1-\gamma)} K_{1}^{1 /(1-\gamma)} d s+M(t, T)^{1 /(1-\gamma)} K_{2}^{1 /(1-\gamma)}\right)
$$

with $M$ as in (25). The claimed upper bound (33) follows from Proposition 4 and that $\gamma \in(0,1)$. The upper bound $(36)$ is obtained in a similar fashion. 
Remark: For aggressive agents, inequality (32) may be directly obtained, without proving a lower bound for $h$, by applying in (5) and (7) the sub-optimality of the zero consumption policy. This automatically yields that the value function of the aggressive agent is bounded from below by its pure investment analogue. This argument, however, fails to yield a finite lower bound for the case of conservative agents.

\section{Appendix B.}

Proof of Proposition 8: The first order conditions in the HJB equation (10) yield that the maxima for the consumption and investment optimal controls occur, respectively, at

$$
C^{*}(x, y, t)=K_{1}^{1 /(1-\gamma)} V_{x}(x, y, t)^{1 /(\gamma-1)}
$$

and

$$
\pi^{*}(x, y, t)=-\frac{\mu(y, t)}{\sigma^{2}(y, t)} \frac{V_{x}(x, y, t)}{V_{x x}(x, y, t)}-\rho \frac{a(y, t)}{\sigma(y, t)} \frac{V_{x y}(x, y, t)}{V_{x x}(x, y, t)} .
$$

Using the value function representation, $V(x, y, t)=\frac{x^{\gamma}}{\gamma} h(y, t)^{1-\gamma}$, we deduce

$$
C^{*}(x, y, t)=K_{1}^{1 /(1-\gamma)} \frac{x}{h(y, t)}
$$

(cf. (38)) and

$$
\pi^{*}(x, y, t)=\frac{\mu(y, t)}{(1-\gamma) \sigma^{2}(y, t)} x+\rho \frac{a(y, t)}{\sigma(y, t)} \frac{h_{y}(y, t)}{h(y, t)} x
$$

(cf. (40) and (41)). The optimality of the feedback pair $\left(C_{s}^{*}, \pi_{s}^{*}\right)$,

$$
C_{s}^{*}=C^{*}\left(X_{s}^{*}, Y_{s}, s\right)=K_{1}^{1 /(1-\gamma)} \frac{X_{s}^{*}}{h\left(Y_{s}, s\right)}
$$

and

$$
\pi_{s}^{*}=\pi^{*}\left(X_{s}^{*}, Y_{s}, s\right)=\frac{\mu\left(Y_{s}, s\right)}{(1-\gamma) \sigma^{2}\left(Y_{s}, s\right)} X_{s}^{*}+\rho \frac{a\left(Y_{s}, s\right)}{\sigma\left(Y_{s}, s\right)} \frac{h_{y}\left(Y_{s}, s\right)}{h\left(Y_{s}, s\right)} X_{s}^{*}
$$

with $X_{s}^{*}$ solving, for $t \leq s \leq T$,

$$
d X_{s}^{*}=\mu\left(Y_{s}, s\right) \pi_{s}^{*} d s-C_{s}^{*} d s+\sigma\left(Y_{s}, s\right) \pi_{s}^{*} d W_{s}^{1}
$$

with $X_{t}^{*}=x$ follows from standard verification arguments (see Theorems IV.3.1 and Corollary IV.3.1 in Fleming and Soner (1993)) under appropriate growth and regularity properties of the market coefficients, and the functions $h$ and $h_{y}$.

Proof of Proposition 10: We first observe that, for $h>0$, the quadratic nonlinear term in (17) can be written as

$$
-\frac{1}{2} \gamma\left(1-\rho^{2}\right) a^{2} \frac{h_{y}^{2}}{h}=\left(1-\rho^{2}\right) \min _{u}\left(\frac{1}{2} u^{2} \frac{\gamma}{(1-\gamma)^{2}} h+u a \frac{\gamma}{1-\gamma} h_{y}\right)
$$

if $\gamma \in(0,1)$ and as

$$
-\frac{1}{2} \gamma\left(1-\rho^{2}\right) a^{2} \frac{h_{y}^{2}}{h}=\left(1-\rho^{2}\right) \max _{u}\left(\frac{1}{2} u^{2} \frac{\gamma}{(1-\gamma)^{2}} h+u a \frac{\gamma}{1-\gamma} h_{y}\right)
$$

if $\gamma \in(-\infty, 0)$. 
For $\gamma \in(0,1)$, the quasilinear equation (17) can be then expressed as a HJB equation associated with a quadratic minimization problem, namely,

$$
\begin{aligned}
& h_{t}+\frac{1}{2} a^{2} h_{y y}+\min _{u}\left(\frac{1}{2}\left(\lambda^{2}+\left(1-\rho^{2}\right) u^{2}\right) \frac{\gamma}{(1-\gamma)^{2}} h\right. \\
& \left.\left(b+a \frac{\gamma}{1-\gamma}\left(\rho \lambda+\left(1-\rho^{2}\right) u\right)\right) h_{y}\right)+K_{1}^{1 /(1-\gamma)}=0
\end{aligned}
$$

with $h(y, T)=K_{2}^{1 /(1-\gamma)}$. Similarly, if $\gamma \in(-\infty, 0)$, equation (17) can be written as

$$
\begin{aligned}
& h_{t}+\frac{1}{2} a^{2} h_{y y}+\max _{u}\left(\frac{1}{2}\left(\lambda^{2}+\left(1-\rho^{2}\right) u^{2}\right) \frac{\gamma}{(1-\gamma)^{2}} h\right. \\
& \left.\left(b+a \frac{\gamma}{1-\gamma}\left(\rho \lambda+\left(1-\rho^{2}\right) u\right)\right) h_{y}\right)+K_{1}^{1 /(1-\gamma)}=0
\end{aligned}
$$

with $h(y, T)=K_{2}^{1 /(1-\gamma)}$.

From the first order conditions, we see that for all agents, the optimum occurs, at least formally, at the point

$$
u^{*}(y, t)=-(1-\gamma) a \frac{h_{y}(y, t)}{h(y, t)} .
$$

We continue the proof for the case $\gamma \in(0,1)$ since the arguments are easily adapted when $\gamma \in(-\infty, 0)$.

We first introduce a stochastic control problem whose value function will be given by $h$. To this end, we consider a probability space $(\Omega, \mathcal{F}, \overline{\mathbb{P}})$ and in it define two standard Brownian motions $\bar{W}^{1}$ and $\bar{W}$ having correlation $\rho$. We also introduce a state controlled diffusion process, say $\bar{Y}$, solving

$$
d \bar{Y}_{s}=\left(b\left(\bar{Y}_{s}, s\right)+a\left(\bar{Y}_{s}, s\right) \frac{\gamma}{1-\gamma}\left(\rho \lambda\left(\bar{Y}_{s}, s\right)+\left(1-\rho^{2}\right) u_{s}\right)\right) d s+a\left(\bar{Y}_{s}, s\right) d \bar{W}_{s} .
$$

Using classical arguments from optimal stochastic control we then see that $h$ can be written as

$$
h(y, t)=\inf _{\mathcal{U}} E_{\overline{\mathbb{P}}}\left(\int_{t}^{T} K_{1}^{1 /(1-\gamma)} N(t, s) d s+K_{2}^{1 /(1-\gamma)} N(t, T) \mid \bar{Y}_{t}=y\right)
$$

where $\mathcal{U}$ is the set of admissible policies appropriately defined and

$$
N(t, s)=\exp \left(\int_{t}^{s} \frac{1}{2}\left(\lambda_{\theta}^{2}+\left(1-\rho^{2}\right) u_{\theta}^{2}\right) \frac{\gamma}{(1-\gamma)^{2}} d \theta\right) .
$$

Using a standard Girsanov's transformation we may express the above expected criterion in terms of the original historical measure $\mathbb{P}$. Indeed, consider the orthogonal decomposition

with

$$
d \bar{W}_{s}=\rho d \bar{W}_{s}^{1}+\sqrt{1-\rho^{2}} d \bar{W}_{s}^{1, \perp}
$$

and

$$
d \bar{W}_{s}^{1}=d W_{s}^{1}+\frac{\gamma}{\gamma-1} \lambda_{s} d s
$$

$$
d \bar{W}_{s}^{1, \perp}=d W_{s}^{1, \perp}+\sqrt{1-\rho^{2}} \frac{\gamma}{\gamma-1} u_{s} d s
$$


Then the Radon-Nikodym derivative $d \overline{\mathbb{P}} / d \mathbb{P}$ takes the form

$$
\begin{aligned}
\frac{d \overline{\mathbb{P}}}{d \mathbb{P}}=\exp & -\int_{0}^{T} \frac{\gamma}{\gamma-1} \lambda_{s} d W_{s}^{1}-\int_{0}^{T} \frac{\gamma}{\gamma-1} \sqrt{1-\rho^{2}} u_{s} d W_{s}^{1, \perp} \\
& \left.-\int_{0}^{T} \frac{1}{2} \frac{\gamma^{2}}{(\gamma-1)^{2}}\left(\lambda_{s}^{2}+\left(1-\rho^{2}\right) u_{s}^{2}\right) d s\right)
\end{aligned}
$$

which allows us to express the criterion

$$
h(y, t)=\inf _{\mathcal{U}} E_{\mathbb{P}}\left(\left.\int_{t}^{T} K_{1}^{1 /(1-\gamma)} \frac{d \overline{\mathbb{P}}}{d \mathbb{P}}\right|_{t} ^{s} N(t, s) d s+\left.K_{2}^{1 /(1-\gamma)} \frac{d \overline{\mathbb{P}}}{d \mathbb{P}}\right|_{t} ^{T} N(t, T) \mid \bar{Y}_{t}=y\right) .
$$

We next observe that $\overline{\mathbb{P}}$ is not a martingale measure. However, it can be expressed in terms of the martingale measure $\mathcal{Q}$ defined through the Radon-Nikodym derivative

$$
\begin{aligned}
\frac{d \mathcal{Q}}{d \mathbb{P}}=\exp & \left(-\int_{0}^{T} \lambda_{s} d W_{s}^{1}-\int_{0}^{T} \sqrt{1-\rho^{2}} u_{s} d W_{s}^{1, \perp}\right. \\
& \left.-\int_{0}^{T} \frac{1}{2}\left(\lambda_{s}^{2}+\left(1-\rho^{2}\right) u_{s}^{2}\right) d s\right) .
\end{aligned}
$$

The measures $\mathcal{Q}$ and $\overline{\mathbb{P}}$ are related by the expression

$$
\left(\frac{d \mathcal{Q}}{d \mathbb{P}}\right)^{\gamma /(\gamma-1)}=N(0, T) \frac{d \overline{\mathbb{P}}}{d \mathbb{P}}
$$

which allows us to rewrite $h$ as a minimization over martingale measures

$h(y, t)=\inf _{\mathcal{Q} \in \mathcal{Q}_{e}} E_{\mathbb{P}}\left(\int_{t}^{T}\left(\left.\frac{d Q}{d \mathbb{P}}\right|_{t} ^{s}\right)^{\gamma /(\gamma-1)} K_{1}^{1 /(1-\gamma)} d s+\left(\left.\frac{d Q}{d \mathbb{P}}\right|_{t} ^{T}\right)^{\gamma /(\gamma-1)} K_{2}^{1 /(1-\gamma)} \mid \mathcal{F}_{t}\right)$

The result follows from $(66)$ and the relation $u^{*}(y, t) \sqrt{1-\rho^{2}}=\lambda^{\perp, *}(y, t)$.

\section{Appendix C.}

Proof of Proposition 12: If we let $\tilde{h}(y, t)=h(y, t) / K_{1}^{1 /(1-\gamma)}$, the optimal consumption, given in (55), can be rewritten as

$$
C_{s}^{*}(x, y, t)=\frac{x}{\tilde{h}(y, t)}
$$

By dividing equation (17) by $K_{1}^{1 /(1-\gamma)}$, we find that $\tilde{h}(y, t)$ solves

$$
\tilde{h}_{t}+\mathcal{L}^{\gamma} \tilde{h}+\frac{\gamma}{2(1-\gamma)^{2}} \lambda^{2} \tilde{h}-\frac{1}{2} \gamma\left(1-\rho^{2}\right) a^{2} \frac{\tilde{h}_{y}^{2}}{\tilde{h}}+1=0
$$

with $\tilde{h}(y, T)=\left(\frac{K_{2}}{K_{1}}\right)^{1 /(1-\gamma)}$. It then follows, from standard comparison results (see, for example Theorem 3.12 in Protter and Weinberger (1967)) that $\tilde{h}(y, t)$ is increasing in the ratio $K_{2} / K_{1}$. Part (i) follows from (68).

Part (ii) is a direct consequence of expression (55). 
To show part (iii), we first observe that if $a, b$ and $\lambda$ are time homogeneous, we may formally differentiate equation (17) with respect to $t$ to obtain

$$
\left\{\begin{array}{l}
h_{t t}+\mathcal{L}^{\gamma} h_{t}+\frac{\gamma}{2(1-\gamma)^{2}} \lambda^{2} h_{t}-\frac{1}{2} a^{2} \gamma\left(1-\rho^{2}\right)\left(\frac{2 h_{y} h_{y t}}{h}-\frac{h_{y}^{2} h_{t}}{h^{2}}\right)=0 \\
h_{t}(y, T)=-K_{1}^{1 /(1-\gamma)}-\frac{1}{2} \gamma^{2} \frac{\gamma}{(1-\gamma)^{2}} K_{2}^{1 /(1-\gamma)}
\end{array}\right.
$$

Assuming that $-{\frac{K_{1}}{K_{2}}}^{1 /(1-\gamma} \leq \frac{1}{2} \lambda^{2} \frac{\gamma}{(1-\gamma)^{2}}$, a comparison result will yield that $h_{t}(y, t) \leq$ 0 and the claim follows from (55).

To establish part (iv), we differentiate equation (17) with respect to $\rho$ to obtain

$$
\left\{\begin{array}{l}
h_{\rho t}+\mathcal{L}^{\gamma} h_{\rho}+\frac{\gamma}{2(1-\gamma)^{2}} \lambda^{2} h_{\rho}+\lambda a \frac{\gamma}{1-\gamma} h_{y}-\frac{1}{2} a^{2} \gamma\left(1-\rho^{2}\right)\left(\frac{2 h_{y} h_{y \rho}}{h}-\frac{h_{y}^{2} h_{\rho}}{h^{2}}\right)+a^{2} \gamma \rho \frac{h_{y}^{2}}{h}=0 \\
h_{\rho}(y, T)=0 .
\end{array}\right.
$$

Putting together the two terms that do not involve the derivative with respect to $\rho$, we obtain,

$$
\lambda a \frac{\gamma}{1-\gamma} h_{y}+a^{2} \gamma \rho \frac{h_{y}^{2}}{h}=\left(\frac{\lambda}{\sigma(1-\gamma)}+\frac{\rho a}{\sigma} \frac{h_{y}}{h}\right) a \sigma \gamma h_{y}=\frac{\pi}{x} a \sigma \gamma h_{y} .
$$

It turns out that if $\lambda \lambda_{y} \geq 0$, then $h_{y}$ has the same sign as $\gamma$ (see the proof of Proposition 14). Therefore, if there are no short sales $(\pi>0)$,

$$
\left\{\begin{array}{l}
h_{\rho t}+\mathcal{L}^{\gamma} h_{\rho}+\frac{\gamma}{2(1-\gamma)^{2}} \lambda^{2} h_{\rho}-\frac{1}{2} a^{2} \gamma\left(1-\rho^{2}\right)\left(\frac{2 h_{y} h_{y \rho}}{h}-\frac{h_{y}^{2} h_{\rho}}{h^{2}}\right) \leq 0 \\
h_{\rho}(y, T)=0
\end{array}\right.
$$

and we easily conclude.

Proof of Proposition 13: Part (i) follows from expression (56), while part (ii) follows from the observation that $h_{y}(y, T)=0$.

Proof of Proposition 14: We prove the result for $\gamma \in(0,1)$ and $\lambda_{y} \lambda>0$, since the argument can be easily adapted to the other cases. Formally differentiating equation (17) with respect to $y$, we obtain

$$
\begin{gathered}
h_{t y}+\mathcal{L}^{\gamma} h_{y}+\lambda \lambda_{y} \frac{\gamma}{(1-\gamma)^{2}} h+\left(b_{y}+\frac{\gamma}{1-\gamma}(\rho a \lambda)_{y}+\frac{1}{2} \frac{\gamma}{(1-\gamma)} \lambda^{2}\right) h_{y} \\
+a a_{y} h_{y y}-\frac{1}{2} a^{2} \gamma\left(1-\rho^{2}\right)\left(\frac{2 h_{y} h_{y y}}{h}-\frac{h_{y}^{3}}{h^{2}}\right)-a a_{y} \gamma\left(1-\rho^{2}\right) \frac{h_{y}^{2}}{h}=0
\end{gathered}
$$

with $h_{y}(y, T)=0$. Since $\frac{\gamma}{(1-\gamma)^{2}} \lambda \lambda_{y} h \geq 0$, we deduce that

$$
\begin{aligned}
h_{t y}+ & \mathcal{L}^{\gamma} h_{y}+\left(b_{y}+\frac{\gamma}{1-\gamma}(\rho a \lambda)_{y}+\frac{1}{2} \frac{\gamma}{(1-\gamma)} \lambda^{2}\right) h_{y}+a a_{y} h_{y y} \\
& -\frac{1}{2} a^{2} \gamma\left(1-\rho^{2}\right)\left(\frac{2 h_{y} h_{y y}}{h}-\frac{h_{y}^{3}}{h^{2}}\right)-a a_{y} \gamma\left(1-\rho^{2}\right) \frac{h_{y}^{2}}{h} \leq 0
\end{aligned}
$$

This in turn yields $h_{y} \geq 0$, and the monotonicity of $C^{*}(x, y, t)$ follows from equation $(55)$. 
Proof of Proposition 15: In the proof of Proposition 14, we established that for aggressive investors, if $\lambda_{y} \lambda>0$, then $h_{y}>0$. Since $h(y, t)$ is always positive, it follows from (57) that, if $\rho>0$, then $H(y, t)>0$. All other cases can be proved in a similar fashion.

Proof of Proposition 16: Recall that in the proof of Proposition 7, we established that, for all investors,

$$
h(y, t) \geq\left(E_{\mathbb{P} \gamma}\left(M(t, T)^{1 / \delta} \mid Y_{t}=y\right)\right)^{\delta /(1-\gamma)}
$$

while for aggressive agents,

$$
h(y, t) \leq E_{\mathbb{P} \gamma}\left(\int_{t}^{T} M(t, s)^{1 /(1-\gamma)} K_{1}^{1 /(1-\gamma)} d s+M(t, T)^{1 /(1-\gamma)} K_{2}^{1 /(1-\gamma)}\right) .
$$

The lower and upper bounds (58) and (59) then follow directly from equation (55). The arguments for part (ii) follow similarly and they are thus omitted.

\section{Appendix D.}

Proof of Proposition 17: Since the linear equation (28) is the sum of the linear equations (61) and (62), we easily obtain that

$$
\tilde{h}(S, t)=\tilde{h}^{c}(S, t)+\tilde{h}^{w}(S, t) .
$$

We also observe that

$$
\tilde{V}^{c}\left(x \frac{\tilde{h}^{c}}{\tilde{h}}, S, t\right)=\frac{x^{\gamma}}{\gamma} \frac{\tilde{h}^{c}}{\tilde{h}^{\gamma}}
$$

and that

$$
\tilde{V}^{w}\left(x \frac{\tilde{h}-\tilde{h}^{c}}{\tilde{h}}, S, t\right)=\frac{x^{\gamma}}{\gamma} \frac{\left(\tilde{h}-\tilde{h}^{c}\right)^{\gamma}\left(\tilde{h}^{w}\right)^{1-\gamma}}{\tilde{h}^{\gamma}} .
$$

A direct calculation then yields (63).

Following the approach of Section 2(iii) by setting $\rho=1$ in expression (56), we obtain

and

$$
\begin{gathered}
\tilde{\pi}^{*}(x, S, t)=\lambda(S, t) \frac{x}{(1-\gamma) \sigma(S, t)}+a(y, t) \frac{\tilde{h}_{S}(S, t)}{\tilde{h}(S, t)} \frac{x}{\sigma(S, t)}, \\
\tilde{\pi}^{w, *}(x, S, t)=\lambda(S, t) \frac{x}{(1-\gamma) \sigma(S, t)}+a(y, t) \frac{\tilde{h}_{S}^{c}(S, t)}{\tilde{h}^{c}} \frac{x}{\sigma(S, t)}
\end{gathered}
$$

$$
\tilde{\pi}^{c, *}(x, S, t)=\lambda(S, t) \frac{x}{(1-\gamma) \sigma(S, t)}+a(y, t) \frac{\tilde{h}_{S}^{w}(S, t)}{\tilde{h}^{w}} \frac{x}{\sigma(S, t)} .
$$

The linearity of the portfolio policies follows directly from (70). Finally, since

$$
\tilde{C}_{s}^{*}(x, S, t)=K_{1}^{1 /(1-\gamma)} \frac{x}{\tilde{h}(S, t)}
$$

and

$$
\tilde{C}_{s}^{c, *}(x, S, t)=K_{1}^{1 /(1-\gamma)} \frac{x}{\tilde{h}^{c}(S, t)},
$$

the identity $x^{c}=x \frac{\tilde{h}^{c}}{\tilde{h}}$ yields the decomposition of the optimal consumption. 
Proof of Proposition 18: We will assume $\gamma \in(0,1)$ and omit the case $\gamma \in$ $(-\infty, 0)$, since the proof is very similar. We recall that

$$
V(x, y, t)=\frac{x^{\gamma}}{\gamma} h(y, t)^{1-\gamma}
$$

and we let

$$
V^{w}(x, y, t)=\frac{x^{\gamma}}{\gamma} h^{w}(y, t)^{1-\gamma}
$$

and

$$
V^{c}(x, y, t)=\frac{x^{\gamma}}{\gamma} h^{c}(y, t)^{1-\gamma}
$$

where $h^{c}$ and $h^{w}$ solve

$$
h_{t}^{c}+\mathcal{L}^{\gamma} h^{c}+\frac{\gamma}{2(1-\gamma)^{2}} \lambda^{2} h^{c}-\frac{1}{2} \gamma\left(1-\rho^{2}\right) a^{2} \frac{\left(h_{y}^{c}\right)^{2}}{h^{c}}+K_{1}^{1 /(1-\gamma)}=0
$$

with $h^{c}(y, T)=0$ and

$$
h_{t}^{w}+\mathcal{L}^{\gamma} h^{w}+\frac{\gamma}{2(1-\gamma)^{2}} \lambda^{2} h^{w}-\frac{1}{2} \gamma\left(1-\rho^{2}\right) a^{2} \frac{\left(h_{y}^{w}\right)^{2}}{h^{w}}=0
$$

with $h^{w}(y, T)=K_{2}^{1 / 1-\gamma}$, respectively.

The proof will then follow if we establish the inequalities

$$
\begin{aligned}
h(y, t)^{1-\gamma} & \geq\left(h^{c}(y, t)+h^{w}(y, t)\right)^{1-\gamma} \\
& \geq k^{\gamma} h^{c}(y, t)^{1-\gamma}+(1-k)^{\gamma} h^{w}(y, t)^{1-\gamma} .
\end{aligned}
$$

The second inequality is Hölder's inequality and holds, as an equality, if and only if

$$
k(y, t)=\frac{h^{c}(y, t)}{h^{c}(y, t)+h^{w}(y, t)} .
$$

To demonstrate the first inequality, we need to show that

$$
h^{*}(y, t)=h^{c}(y, t)+h^{w}(y, t)
$$

is a subsolution of equation (17). In other words, we need to show that

$$
\left\{\begin{array}{l}
h_{t}^{c}+h_{t}^{w}+\mathcal{L}^{\gamma} h^{c}+\mathcal{L}^{\gamma} h^{w}+\frac{\gamma}{2(1-\gamma)^{2}} \lambda^{2} h^{c}+\frac{\gamma}{2(1-\gamma)^{2}} \lambda^{2} h^{w} \\
-\frac{1}{2} a^{2} \gamma\left(1-\rho^{2}\right) \frac{\left(h_{y}^{c}+h_{y}^{w}\right)^{2}}{h^{c}+h^{w}}+K_{1}^{1 /(1-\gamma} \geq 0 \\
h^{c}(y, T)+h^{w}(y, T)=K_{2}^{1 /(1-\gamma)} .
\end{array}\right.
$$

Note that $h^{c}$ solves

$$
h_{t}^{c}+\mathcal{L}^{\gamma} h^{c}+\frac{\gamma}{2(1-\gamma)^{2}} \lambda^{2} h^{c}-\frac{1}{2} \gamma\left(1-\rho^{2}\right) a^{2} \frac{\left(h_{y}^{c}\right)^{2}}{h^{c}}+K_{1}^{1 /(1-\gamma)}=0
$$

with $h^{c}(y, T)=0$, and that $h^{w}$ solves

$$
h_{t}^{w}+\mathcal{L}^{\gamma} h^{w}+\frac{\gamma}{2(1-\gamma)^{2}} \lambda^{2} h^{w}-\frac{1}{2} \gamma\left(1-\rho^{2}\right) a^{2} \frac{\left(h_{y}^{w}\right)^{2}}{h^{w}}=0
$$

with $h^{w}(y, T)=K_{2}^{1 / 1-\gamma}$. 
Applying (75) and (76) to (74), we obtain that $h^{*}(y, t)$ is a subsolution of equation (17) if and only if

$$
-\frac{1}{2} a^{2} \gamma\left(1-\rho^{2}\right)\left[\frac{\left(h_{y}^{c}+h_{y}^{w}\right)^{2}}{h^{c}+h^{w}}-\frac{\left(h_{y}^{c}\right)^{2}}{h^{c}}-\frac{\left(h_{y}^{w}\right)^{2}}{h^{w}}\right] \geq 0
$$

or, equivalently, if and only if

$$
\frac{1}{2} a^{2} \gamma\left(1-\rho^{2}\right)\left[\frac{\left(h_{y}^{c} h^{w}-h_{y}^{w} h^{c}\right)^{2}}{\left(h^{c}+h^{w}\right) h^{c} h^{w}}\right] \geq 0 .
$$

From this expression, we conclude that $h(y, t) \geq h^{*}(y, t)$, with equality holding if and only if (i) $a=0$, (ii) $\rho= \pm 1$, or (iii) $h_{y}^{c} h^{w}-h_{y}^{w} h^{c}=0$.

The first two cases correspond to a complete market setting, and the last case leads us to a contradiction. Indeed, if $h_{y}^{c} h^{w}-h_{y}^{w} h^{c}=0$, the expression $\frac{h^{w}}{h^{c}}$ is not a function of $y$. This implies that

$$
k(y, t)=\frac{h^{c}(y, t)}{h^{c}(y, t)+h^{w}(y, t)}=\frac{1}{1+\frac{h^{w}}{h^{c}}}
$$

is a pure function of $t$, say $k(t)$. Substituting $h(y, t)=\frac{h^{c}(y, t)}{k(t)}$ into equation (17) yields

$$
-\frac{h^{c} k_{t}}{k^{2}}+\frac{1}{k}\left(h_{t}^{c}+\mathcal{L}^{\gamma} h^{c}+\frac{\gamma}{2(1-\gamma)^{2}} \lambda^{2} h^{c}-\frac{1}{2} a^{2} \gamma\left(1-\rho^{2}\right) \frac{h_{y}^{c^{2}}}{h^{c}}\right)+K_{1}^{1 /(1-\gamma)}=0
$$

which simplifies to

$$
\left\{\begin{array}{l}
k_{t}=\frac{k^{2}-k}{h^{c}(y, t)} K_{1}^{1 /(1-\gamma)} \\
k(T)=0
\end{array}\right.
$$

Therefore $k(t)=0 \forall t \in[0, T]$. Using (77) implies that $V(x, y, t)=V^{w}(x, y, t)$, which can only be true if the investor derives no utility from consumption. 